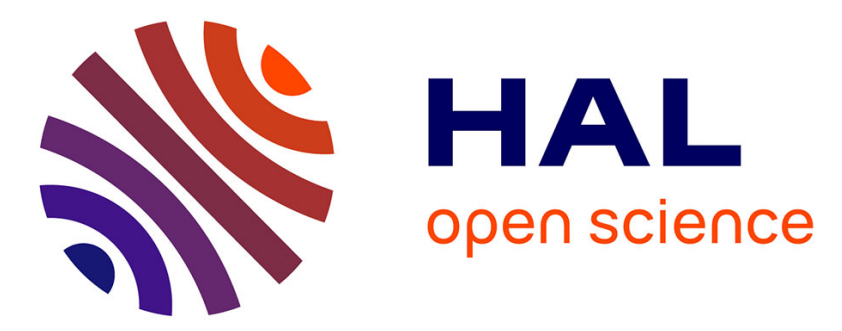

\title{
On an Optional Semimartingale Decomposition and the Existence of a Deflator in an Enlarged Filtration
}

Anna Aksamit, Tahir Choulli, Monique Jeanblanc

\section{To cite this version:}

Anna Aksamit, Tahir Choulli, Monique Jeanblanc. On an Optional Semimartingale Decomposition and the Existence of a Deflator in an Enlarged Filtration. Séminaire de Probabilités, 2015, 10.1007/978-3-319-18585-9_9. hal-01250968

\section{HAL Id: hal-01250968 \\ https://hal.science/hal-01250968}

Submitted on 5 Jan 2016

HAL is a multi-disciplinary open access archive for the deposit and dissemination of scientific research documents, whether they are published or not. The documents may come from teaching and research institutions in France or abroad, or from public or private research centers.
L'archive ouverte pluridisciplinaire HAL, est destinée au dépôt et à la diffusion de documents scientifiques de niveau recherche, publiés ou non, émanant des établissements d'enseignement et de recherche français ou étrangers, des laboratoires publics ou privés. 


\title{
On an optional semimartingale decomposition and the existence of a deflator in an enlarged filtration
}

Anna Aksamit, Tahir Choulli and Monique Jeanblanc

\begin{abstract}
Given a reference filtration $\mathbb{F}$, we consider the cases where an enlarged filtration $\mathbb{G}$ is constructed from $\mathbb{F}$ in two different ways: progressively with a random time or initially with a random variable. In both situations, under suitable conditions, we present a $\mathbb{G}$-optional semimartingale decomposition for $\mathbb{F}$-local martingales. Our study is then applied to answer the question of how an arbitrage-free semimartingale model is affected when stopped at the random time in the case of progressive enlargement or when the random variable used for initial enlargement satisfies Jacod's hypothesis. More precisely, we focus on the No-Unbounded-Profit-with-BoundedRisk (NUPBR) condition. We provide alternative proofs of some results from [5], with a methodology based on our optional semimartingale decomposition, which reduces significantly the length of the proof.
\end{abstract}

\section{Introduction}

We are interested with some specific enlargements of a given filtration, namely the progressive one and the initial one. The progressive enlargement $\mathbb{G}$ of a filtration $\mathbb{F}$ with a random time (a positive random variable) $\tau$, is the smallest filtration larger than $\mathbb{F}$ making $\tau$ a stopping time. It is known that any $\mathbb{F}$-martingale, stopped at time $\tau$ is a $\mathbb{G}$ semi-martingale. In this paper, we do not consider the behavior of

Anna Aksamit

Laboratoire de Mathématiques et Modélisation d'Évry (LaMME), Université d'Évry-Vald'Essonne, UMR CNRS 8071, e-mail: ania.aksamit@gmail.com

Tahir Choulli

Mathematical and Statistical Sciences Department, University of Alberta, Edmonton, Canada, email: tchoulli@ualberta.ca

Monique Jeanblanc

Laboratoire de Mathématiques et Modélisation d'Évry (LaMME), Université d'Évry-Vald'Essonne, UMR CNRS 8071, e-mail: monique.jeanblanc@univ-evry.fr 
$\mathbb{F}$-martingales after $\tau$, which is presented in [3], and requires specific assumptions on the random time $\tau$.

Then, we study the case where the enlarged filtration $\mathbb{G}$ is constructed from $\mathbb{F}$ as an initial enlargement, that is, adding to all the elements $\mathscr{F}_{t}$ of the filtration $\mathbb{F}$ a random variable $\xi$. We focus on a specific situation where the hypothesis $\left(\mathscr{H}^{\prime}\right)$, i.e., the property that each $\mathbb{F}$-martingale remains a $\mathbb{G}$-semimartingale, is satisfied. More precisely, we shall assume that the $\mathbb{F}$-conditional law of $\xi$ is absolutely continuous with respect to the unconditional law of $\xi$ (Jacod's hypothesis, see Definition1 below).

The goal of the paper is to study the impact of the new information for arbitrage opportunities in a financial market: assuming that one deals with an arbitrage free financial market with $\mathbb{F}$-adapted prices, can an agent using $\mathbb{G}$-adapted strategies realize arbitrage opportunities? More precisely we study how the No-UnboundedProfit-with-Bounded-Risk (NUPBR) condition (see Definition 3 below) will be preserved in the enlarged filtration. This condition is closely related with the notion of log-optimal portfolios and optimal growth rate portfolio. A general study of the NUPBR condition, and a list of references on the subject can be found in Kabanov et al. [22].

The literature on arbitrage conditions in an enlarged filtration is important, even if the subject is not so popular in mathematical finance. Quite surprisingly, the hypothesis that all the investors have the same knowledge is usually done in the literature, even if this hypothesis is not satisfied in reality. The main difficulty is that it is not easy to compare stochastic processes in various filtration (the most common approach is filtering study). Here we are interested with the opposite direction: some investor has an information larger than the one generated by prices of asset he is willing to trade. For progressive enlargement, the case of classical arbitrages is presented in [11], and it is proved that, for a class of random times (called honest times) arbitrages can occur in the case where the market described in the filtration $\mathbb{F}$ is complete and arbitrage free (see also [15] for the Brownian case). However, to the best of our knowledge, no necessary and sufficient conditions are known in an incomplete model. The recent literature concerns a weaker notion of arbitrages, called No-Unbounded-Profit-with-Bounded-Risk (NUPBR), deeply related with optimization problems, see [7]. A first paper on that subject was [11], in which the authors are dealing with continuous processes. Many examples of progressive enlargement (in particular for discontinuous processes) are given in [2]. A general study, giving necessary and sufficient condition for the stability of NUPBR condition is presented in [5]. A different proof of some results of that paper (mainly sufficient conditions), based on another representation of the deflators (see subsection 1.3 for definition), is given in [1]. We shall explain here how our results are linked with the ones in [1]. The recent paper of Song [33] contains also a study of deflator in a progressive enlargement setting.

The case of initial enlargement was studied under the name of insider trading. Many papers, including $[12,13]$ and the thesis [6] present results under an assump- 
tion stronger that the absolute continuity Jacod's hypothesis.

In the first section, we recall some basic definitions and results on enlargement of filtration and on arbitrage opportunities. Section 2 addresses the case of progressive enlargement with $\tau$ and $\mathbb{F}$-martingales stopped at $\tau$. In subsection 2.1, we introduce a particular optional semi-martingale decomposition, which will be useful in the sequel, and we give the link between this decomposition and the deflator exhibited in the literature (see [1] and [5]). In subsection 2.2, we provide alternative and shorter proofs of some results from [5], and give a condition so that the NUPBR condition is preserved, using a methodology different from the one used in [5] avoiding the introduction of optional integral, and based on our optional semimartingale decomposition.

Section 3 presents the case of initial enlargement. In subsection 3.2, we give an optional decomposition result for the $\mathbb{F}$-martingales, when the added random variable satisfies Jacod's hypothesis.We also obtain a result concerning the relationship between the predictable brackets of semimartingale computed in both filtrations. Then, we address the question of stability of the NUPBR condition. The results presented in this last section were obtained in parallel and independently of [1].

The last section 4 presents a link between our optional decomposition and absolutely continuous change of measures.

\section{Preliminaries}

Let $(\Omega, \mathscr{G}, \mathbb{P})$ be a complete probability space and $\mathbb{F}=\left(\mathscr{F}_{t}\right)_{t \geq 0}$ be a filtration satisfying the usual conditions. We say that a filtration $\mathbb{G}=\left(\mathscr{G}_{t}\right)_{t \geq 0}$ is an enlargement of $\mathbb{F}$ if, for each $t \geq 0$, we have $\mathscr{F}_{t} \subset \mathscr{G}_{t}$.

We recall some standard definitions and set some notation. For a filtration $\mathbb{H}$, the optional $\sigma$-field on $\Omega \times \mathbb{R}_{+}$, denoted by $\mathscr{O}(\mathbb{H})$, is the $\sigma$-field generated by all càdlàg $\mathbb{H}$-adapted processes and the predictable $\sigma$-field on $\Omega \times \mathbb{R}_{+}$, denoted by $\mathscr{P}(\mathbb{H})$, is the $\sigma$-field generated by all left-continuous $\mathbb{H}$-adapted processes. A stochastic set or process is called $\mathbb{H}$-optional (respectively $\mathbb{H}$-predictable) if it is $\mathscr{O}(\mathbb{H})$-measurable (respectively $\mathscr{P}(\mathbb{H})$-measurable).

For an $\mathbb{H}$-semimartingale $Y$, the set of $\mathbb{H}$-predictable processes integrable with respect to $Y$ is denoted by $L(Y, \mathbb{H})$ and for $H \in L(Y, \mathbb{H})$, we denote by $H$. $Y$ the stochastic integral $\int_{0}^{\cdot} H_{S} d Y_{s}$.

As usual, for a process $X$ and a random time $\vartheta$, we denote by $X^{\vartheta}$ the stopped process. For a given semimartingale $X, \mathscr{E}(X)$ stands for the stochastic exponential of $X$. The continuous local martingale part and the jump process of a càdlàg semimartingale $X$ are denoted respectively by $X^{c}$ and $\Delta X$. 


\subsection{Progressively enlarged filtration}

Consider a random time $\tau$, i.e., a positive random variable. Then, we define two $\mathbb{F}$ supermartingales, which are the corner stone for the classical enlargement decomposition formulae (2) and (3), in a progressive enlargement framework (see [19]), given by

$$
Z_{t}:=\mathbb{P}\left(\tau>t \mid \mathscr{F}_{t}\right) \quad \text { and } \quad \widetilde{Z}_{t}:=\mathbb{P}\left(\tau \geq t \mid \mathscr{F}_{t}\right)
$$

In other terms, $Z$ is the optional projection of $\mathbb{1}_{[0, \tau[}$, whereas $\widetilde{Z}$ is the optional projection of $\mathbb{1}_{[0, \tau]}$. Let $A^{o}$ be the $\mathbb{F}$-dual optional projection of the increasing process $A:=\mathbb{1}_{[\tau, \infty[} ;$ then (see [19]), the process

$$
m:=Z+A^{o}
$$

is an $\mathbb{F}$-martingale. Furthermore, $\widetilde{Z}=Z_{-}+\Delta m$.

We denote by $\mathbb{F}^{\tau}=\left(\mathscr{F}_{t}^{\tau}\right)_{t \geq 0}$ the right-continuous progressively enlarged filtration with the random time $\tau$, i.e.,

$$
\mathscr{F}_{t}^{\tau}:=\bigcap_{s>t}\left(\mathscr{F}_{s} \vee \sigma(\tau \wedge s)\right)
$$

The following result from [20] states that any $\mathbb{F}$-local martingale stopped at $\tau$ is an $\mathbb{F}^{\tau}$-semimartingale.

Proposition 1. Let $X$ be an $\mathbb{F}$-local martingale. Then, $X^{\tau}$ is an $\mathbb{F}^{\tau}$-semimartingale which can be decomposed as

$$
X_{t}^{\tau}=\widehat{X}_{t}+\int_{0}^{t \wedge \tau} \frac{1}{Z_{s-}} d\langle X, m\rangle_{s}^{\mathbb{F}}
$$

where $\widehat{X}$ is an $\mathbb{F}^{\tau}$-local martingale.

In what follows, we will refer to the equality (2) as the predictable decomposition of the $\mathbb{F}^{\tau}$-semi-martingale $X^{\tau}$.

Remark 1. The decomposition (2) contains a predictable bracket computed in $\mathbb{F}$. When working in a larger filtration $\mathbb{G}$, predictable brackets are computed in $\mathbb{G}$. As can be seen in [5], we are faced to the problem of comparison of the two different brackets.

Remark 2. It is rather easy to check that $Z_{-}$does not vanishes on the set $\{t \leq \tau\}$. However, the first time where this process vanishes will play an important rôle.

Remark 3. Using the $\mathbb{F}$-local martingale

$$
N:=\mathscr{E}\left(\frac{1}{Z_{-}} \mathbb{1}_{\left\{Z_{-}>0\right\}} \cdot m\right),
$$

Kardaras [25] notes that the decomposition (2) can be written $X_{t}^{\tau}=\widehat{X}_{t}+\int_{0}^{t \wedge \tau} \frac{1}{N_{s-}} d\langle X, N\rangle_{s}^{\mathbb{F}}$. 


\subsection{Initially enlarged filtration}

Let $\xi$ be a random variable valued ${ }^{1}$ in $(\mathbb{R}, \mathscr{B})$.

Definition 1. The random variable $\xi$ satisfies the absolute continuity Jacod's hypothesis if there exists a $\sigma$-finite positive measure $\eta$ on $(\mathbb{R}, \mathscr{B})$ such that for every $t \geq 0$,

$$
\mathbb{P}\left(\xi \in d u \mid \mathscr{F}_{t}\right)(\omega) \ll \eta(d u), \mathbb{P}-\text { a.s.. }
$$

As shown by Jacod [17], without loss of generality, $\eta$ can be taken as the law of $\xi$. We do not impose any condition on $\eta$, in particular, it is not necessarily atomless. The random variable $\xi$ satisfies the equivalence Jacod's hypothesis if

$$
\mathbb{P}\left(\xi \in d u \mid \mathscr{F}_{t}\right)(\omega) \sim \eta(d u), \mathbb{P}-\text { a.s.. }
$$

Let $\mathbb{F}^{\sigma(\xi)}=\left(\mathscr{F}^{\sigma(\xi)}\right)_{t \geq 0}$ be the right-continuous initial enlargement of the filtration $\mathbb{F}$ with the random variable $\xi$, i.e.,

$$
\mathscr{F}_{t}^{\sigma(\xi)}:=\bigcap_{s>t}\left(\mathscr{F}_{s} \vee \sigma(\xi)\right) .
$$

The following result is due to Jacod [17, Lemme 1.8]. We give here the formulation of Amendinger as it provides a nice measurability property (see [6, Remark 1 , page 17]).

Proposition 2. For $\xi$ satisfying the absolute continuity Jacod's hypothesis, there exists a non negative $\mathscr{O} \otimes \mathscr{B}$-measurable function $(t, \omega, u) \rightarrow q_{t}^{u}(\omega)$ càdlàg in $t$ such that

(i) for every $u, \eta$ a.s., the process $\left(q_{t}^{u}, t \geq 0\right)$ is an $\mathbb{F}$-martingale, and if the stopping time $R^{u}$ is defined as

$$
R^{u}:=\inf \left\{t: q_{t-}^{u}=0\right\}
$$

one has $q^{u}>0$ and $q_{-}^{u}>0$ on $\llbracket 0, R^{u}\left[\right.$ and $q^{u}=0$ on $\llbracket R^{u}, \infty \llbracket$,

(ii) for every $t \geq 0$, the measure $q_{t}^{u}(\omega) \eta(d u)$ is a version of $\mathbb{P}\left(\xi \in d u \mid \mathscr{F}_{t}\right)(\omega)$.

It is rather clear that we shall have to deal with the family of processes $\left(q^{u}, u \in \mathbb{R}\right)$, that we shall call parametrized processes.

Definition 2. Consider a mapping $X:(t, \omega, u) \rightarrow X_{t}^{u}(\omega)$ on $\mathbb{R}_{+} \times \Omega \times \mathbb{R}$ with values in $\mathbb{R}$. Let $\mathscr{J}$ be a class of $\mathbb{F}$-optional processes, for example the class of $\mathbb{F}$-(local) martingales or the class of $\mathbb{F}$-locally integrable variation processes. Then, $\left(X^{u}, u \in\right.$ $\mathbb{R}$ ) is called a parametrized $\mathscr{J}$-process if for each $u \in \mathbb{R}$ the process $X^{u}$ belongs to $\mathscr{J}$ and if it is measurable with respect to $\mathscr{O}(\mathbb{F}) \otimes \mathscr{B}$.

By [34, Proposition 3], the second condition can be obtained by taking appropriate versions of processes $X^{u}$.

${ }^{1}$ The random variable $\xi$ can take values in a more general space without any difficulty. 
The next theorem gives, in the case of equivalence Jacod's hypothesis, a particular change of measure making the reference filtration $\mathbb{F}$ and the random variable $\xi$ independent, see [32], [6, Proposition 1.6], [12].

Theorem 1. Assume that the equivalence Jacod's hypothesis is satisfied, so that $\mathbb{P}\left(\xi \in d u \mid \mathscr{F}_{t}\right)=q_{t}^{u} \eta(d u)$ with $q_{t}^{u}>0,(\eta \otimes P)$ a.s. . Then

(a) the process $\frac{1}{q^{\xi}}$ is an $\mathbb{F}^{\sigma(\xi)}$-martingale,

(b) the probability measure $\mathbb{P}^{*}$, defined as

$$
\left.\frac{d \mathbb{P}^{*}}{d \mathbb{P}}\right|_{\mathscr{F}_{t}(\xi)}=\frac{1}{q_{t}^{\xi}}
$$

has the following properties:

(i) under $\mathbb{P}^{*}, \tau$ is independent from $\mathscr{F}_{t}$ for any $t \geq 0$,

(ii) $\left.\mathbb{P}^{*}\right|_{\mathscr{F}_{t}}=\left.\mathbb{P}\right|_{\mathscr{F}_{t}}$,

(iii) $\left.\mathbb{P}^{*}\right|_{\sigma(\xi)}=\left.\mathbb{P}\right|_{\sigma(\xi)}$.

Remark 4. Note that, under Jacod's equivalence hypothesis, if the price process $S$ is such that there are no arbitrages in $\mathbb{F}$, then there are no arbitrages in $\mathbb{G}$. Indeed, taking $\mathbb{P}$ as an equivalent martingale measure in $\mathbb{F}$, the previous result proves that $\mathbb{P}^{*}$ is an equivalent martingale measure in $\mathbb{G}$.

We now recall the computation of $\mathbb{F}$-predictable and $\mathbb{F}$-optional projections of $\mathbb{F}^{\sigma(\xi)}$-adapted processes. The first part is due to Jacod [17, Lemme 1.10], the second part is found in Amendinger [6, Lemma 1.3].

Lemma 1. Assume that the absolute continuity Jacod's hypothesis is satisfied. (i) Assume that the map $(t, \omega, u) \rightarrow Y_{t}^{u}(\omega)$ is $\mathscr{P}(\mathbb{F}) \otimes \mathscr{B}$-measurable, positive or bounded. Then, the $\mathbb{F}$-predictable projection of the process $\left(Y_{t}^{\xi}\right)_{t \geq 0}$ is given by

$$
p, \mathbb{F}\left(Y^{\xi}\right)_{t}=\int_{\mathbb{R}} Y_{t}^{u} q_{t-}^{u} \eta(d u) \quad t \geq 0
$$

(ii) Assume that the map $(t, \omega, u) \rightarrow Y_{t}^{u}(\omega)$ is $\mathscr{O}(\mathbb{F}) \otimes \mathscr{B}$-measurable, positive or bounded. Then, the $\mathbb{F}$-optional projection of the process $\left(Y_{t}^{\xi}\right)_{t \geq 0}$ is given by

$$
{ }^{o, \mathbb{F}}\left(Y^{\xi}\right)_{t}=\int_{\mathbb{R}} Y_{t}^{u} q_{t}^{u} \eta(d u) \quad t \geq 0 .
$$

As noticed in Jacod [17, Corollary 1.11], Lemma 1 implies in particular that

$$
R^{\xi}=\infty \quad \mathbb{P}-\text { a.s. }
$$

where $R^{u}$ is defined through (4), or equivalently $q_{t}^{\xi}>0$ and $q_{t-}^{\xi}>0$, for $t \geq 0, \mathbb{P}$-a.s. Therefore, the $\mathbb{F}^{\sigma(\xi)}$-optional process $\left(\frac{1}{q_{t}^{\xi}}, t \geq 0\right)$ is well-defined.

The $\mathbb{F}^{\sigma(\xi)}$-semimartingale predictable decomposition of an $\mathbb{F}$-local martingale is given in [17, Theorem 2.5] in the following way: 
Proposition 3. Let $X$ be an $\mathbb{F}$-local martingale. Then, under absolute continuity Jacod's hypothesis

$$
X_{t}=\widehat{X}_{t}+\left.\int_{0}^{t} \frac{1}{q_{s-}^{\xi}} d\left\langle X, q^{u}\right\rangle_{s}^{\mathbb{F}}\right|_{u=\xi}
$$

where $\widehat{X}$ is an $\mathbb{F}^{\sigma(\xi)}$-local martingale.

To ensure the existence of well measurable versions of dual projections of parametrized processes, we assume from now on that the space $L^{1}(\Omega, \mathscr{G}, \mathbb{P})$ is separable. Then, we apply [34, Proposition 4].

In the next proposition, we extend Proposition 3 to a class of parametrized $\mathbb{F}$ local martingales.

Proposition 4. Assume absolute continuity Jacod's hypothesis. Let $\left(X^{u}, u \in \mathbb{R}\right)$ be a parametrized $\mathbb{F}$-local martingale. Then

$$
X_{t}^{\xi}=\widehat{X}_{t}^{\xi}+\left.\int_{0}^{t} \frac{1}{q_{s-}^{\xi}} d\left\langle X^{u}, q^{u}\right\rangle_{s}^{\mathbb{F}}\right|_{u=\xi}
$$

where $\widehat{X}^{\xi}$ is an $\mathbb{F}^{\sigma(\xi)}$-local martingale.

Proof. Let $X$ be of the form $X_{t}^{u}(\omega)=g(u) x_{t}(\omega)$ where $x$ is an $\mathbb{F}$-martingale and $g$ is a Borel function. Then, $X^{\xi}=g(\xi) x$ and, using Jacod's decomposition given in Proposition 3, for $t \geq s$, we have

$$
\begin{aligned}
\mathbb{E}\left(X_{t}^{\xi}-X_{s}^{\xi} \mid \mathscr{F}_{s}^{\sigma(\xi)}\right) & =g(\xi) \mathbb{E}\left(x_{t}-x_{s} \mid \mathscr{F}_{s}^{\sigma(\xi)}\right) \\
& =g(\xi) \mathbb{E}\left(\left.\int_{s}^{t} \frac{1}{q_{v-}^{\xi}} d\left\langle x, q^{u}\right\rangle_{v}\right|_{u=\xi} \mid \mathscr{F}_{s}^{\sigma(\xi)}\right) \\
& =\mathbb{E}\left(\left.\int_{s}^{t} \frac{1}{q_{v-}^{\xi}} d\left\langle X^{u}, q^{u}\right\rangle_{v}\right|_{u=\xi} \mid \mathscr{F}_{s}^{\sigma(\xi)}\right) .
\end{aligned}
$$

For a general $X$, we proceed by Monotone Class Theorem.

\subsection{Local martingale deflators and related notions}

As announced before, we shall study the No Unbounded Profit with Bounded Risk (NUPBR) condition of no arbitrages. We start with some definitions for a general filtration $\mathbb{H}$.

Definition 3. (a) Let $\left(X^{u}, u \in \mathbb{R}\right)$ be a parametrized $\mathbb{H}$-semimartingale. We say that $\left(X^{u}, u \in \mathbb{R}\right)$ satisfies No Unbounded Profit with Bounded Risk condition in the filtration $\mathbb{H}$ (we shall write $\operatorname{NUPBR}(\mathbb{H})$ ) if for each $T<\infty$, the set $\mathscr{K}_{T}(X)$ defined as 


$$
\mathscr{K}_{T}(X):=\left\{\left(H \cdot X^{u}\right)_{T}: H \in L\left(\mathbb{H}, X^{u}\right), H \cdot X^{u} \geq-1 \text { and } u \in \mathbb{R}\right\}
$$

is bounded in probability.

(b) A process $Y$ is called an $\mathbb{H}$-local martingale deflator for $\left(X^{u}, u \in \mathbb{R}\right)$ if it is a strictly positive $\mathbb{H}$-local martingale such that $\left(Y X^{u}, u \in \mathbb{R}\right)$ is a parametrized $\mathbb{H}-\sigma$ martingale.

(c) A process $\widetilde{Y}$ is called an $\mathbb{H}$-supermartingale deflator for $\left(X^{u}, u \in \mathbb{R}\right)$ if it is a strictly positive $\mathbb{H}$-supermartingale such that for each $H \in L\left(\mathbb{H}, X^{u}\right)$ with $H \cdot X^{u} \geq$ -1 , the process $\left(1+H \cdot X^{u}\right) \widetilde{Y}$ is an $\mathbb{H}$-supermartingale.

As proved in [29] in full generality, condition (a) and the existence of a supermartingale deflator stated in Definition 3(c) are equivalent. Moreover, as shown in [31], for a process which does not depend on a parameter, condition (a) and the existence of a local martingale deflator are equivalent. So, we have the following theorem:

Theorem 2. For a semimartingale $X$, the NUPBR condition is equivalent to the existence of a local martingale deflator which is equivalent to the existence of a supermartingale deflator.

The following proposition is a parametrized version of [5, Proposition 2.5].

Proposition 5. Let $\left(X^{u}, u \in \mathbb{R}\right)$ be a parametrized $\mathbb{H}$-adapted semi-martingale. Then, the following assertions are equivalent.

(a) The process $\left(X^{u}, u \in \mathbb{R}\right)$ admits an $\mathbb{H}$-local martingale deflator.

(b) There exist a $\mathscr{P}(\mathbb{H}) \otimes \mathscr{B}$-measurable parametrized process $\left(\phi^{u}, u \in \mathbb{R}\right)$ such that $0<\phi^{u} \leq 1$ and an $\mathbb{H}$-local martingale deflator for $\left(\phi^{u} . X^{u}, u \in \mathbb{R}\right)$.

(c) There exists a sequence of $\mathbb{H}$-stopping times $\left(T_{n}\right)_{n \geq 1}$ that increases to $\infty$ such that for each $n \geq 1$, there exist a probability $\mathbb{Q}_{n}$ on $\left(\Omega, \mathscr{F}_{T_{n}}\right)$ such that $\mathbb{Q}_{n} \sim \mathbb{P}$ and an $\mathbb{H}$-local martingale deflator for $\left(\left(X^{u}\right)^{T_{n}}, u \in \mathbb{R}\right)$ under $\mathbb{Q}_{n}$.

The NUPBR condition is related to other no arbitrage conditions like No Free Lunch with Vanishing Risk (NFLVR) or (classical) No Arbitrage, see [8, 21]; in particular the NUPBR condition is equivalent to both NFLVR condition and No arbitrages condition. However, the NUPBR condition is proved to be an appropriate condition to study some financial notions like numéraire portfolio, or market viability (see $[7,14,23,24,26,27,30]$ and the references therein).

\section{Progressive Enlargement up to a Random Time}

\subsection{Optional semimartingale decomposition for progressive enlargement}

In this section, we derive an $\mathbb{F}^{\tau}$-semimartingale decomposition of any $\mathbb{F}$-local martingale stopped at $\tau$, different from the one given in Proposition 1. Let us start by 
the definition of an important $\mathbb{F}$-stopping time, namely

$$
R:=\inf \left\{t \geq 0: Z_{t}=0\right\}
$$

and define the $\mathbb{F}$-stopping time $\widetilde{R}$ as

$$
\widetilde{R}:=R_{\left\{\widetilde{Z}_{R}=0<Z_{R-}\right\}}=R \mathbb{1}_{\left\{\widetilde{Z}_{R}=0<Z_{R-}\right\}}+\infty \mathbb{1}_{\left\{\widetilde{Z}_{R}=0<Z_{R-}\right\}^{c}}
$$

We establish an optional decomposition in the following theorem. By optional decomposition, we mean that we write a semi-martingale as a martingale plus an optional bounded variation process.

Theorem 3. Let $X$ be an $\mathbb{F}$-local martingale. Then the process

$$
\bar{X}_{t}:=X_{t}^{\tau}-\int_{0}^{t \wedge \tau} \frac{1}{\widetilde{Z}_{s}} d[X, m]_{s}+\left(\Delta X_{\widetilde{R}} \mathbb{1}_{[\widetilde{R}, \infty[}\right)_{t \wedge \tau}^{p, \mathbb{F}}
$$

is an $\mathbb{F}^{\tau}$-local martingale.

Proof. First of all, let us recall that for any $\mathbb{F}$-local martingale, the stopped process $X^{\tau}$ is an $\mathbb{F}^{\tau}$-semimartingale as stated in Proposition 2. Let $H$ be an $\mathbb{F}^{\tau}$-predictable bounded process. Then, there exists an $\mathbb{F}$-predictable bounded process $J$ such that $H \mathbb{1}_{[0, \tau]}=J \mathbb{1}_{[0, \tau]}$. By [19, Section IV-3 and Lemme $\left.(5,17)\right]$, the $\mathbb{F}$-martingale $m$ given in (1) satisfies that for each $H^{1}(\mathbb{F})$ martingale $Y$, one has $\mathbb{E}\left(Y_{\tau}\right)=\mathbb{E}\left([m, Y]_{\infty}\right)$. Thus, we have

$$
\begin{aligned}
\mathbb{E}\left(\left(H \cdot X^{\tau}\right)_{\infty}\right) & \left.=\mathbb{E}\left((J \cdot X)_{\tau}\right)\right)=\mathbb{E}\left([J \cdot X, m]_{\infty}\right) \\
& =\mathbb{E}\left(\int_{0}^{\infty} \frac{J_{s} \widetilde{Z}_{s}}{\widetilde{Z}_{s}} \mathbb{1}_{\left\{\widetilde{Z}_{s}>0\right\}} d[X, m]_{s}\right)+\mathbb{E}\left(\int_{0}^{\infty} J_{s} \mathbb{1}_{\left\{\widetilde{Z}_{s}=0<Z_{s-}\right\}} d[X, m]_{s}\right)
\end{aligned}
$$

Since $\left\{\widetilde{Z}=0<Z_{-}\right\}=[\widetilde{R}]$ and $\Delta m_{\widetilde{R}}=-Z_{\widetilde{R}-}$ on $\{\widetilde{R}<\infty\}$, we can write

$$
\mathbb{1}_{\left\{\widetilde{Z}=0<Z_{-}\right\}} \cdot[X, m]=\Delta X_{\widetilde{R}} \Delta m_{\widetilde{R}} \mathbb{1}_{[\widetilde{R}, \infty[}=-Z_{\widetilde{R}-} \Delta X_{\widetilde{R}} \mathbb{1}_{[\widetilde{R}, \infty[} .
$$

Furthermore, due to the fact that $J$ and $[X, m]$ are $\mathbb{F}$-adapted, we obtain

$$
\mathbb{E}\left(\left(H \cdot X^{\tau}\right)_{\infty}\right)=\mathbb{E}\left(\int_{0}^{\tau} \frac{J_{s}}{\widetilde{Z}_{s}} \mathbb{1}_{\left\{\widetilde{Z}_{s}>0\right\}} d[X, m]_{s}\right)-\mathbb{E}\left(\int_{0}^{\infty} J_{s} Z_{s-} d\left(\Delta X_{\widetilde{R}} \mathbb{1}_{[\widetilde{R}, \infty[}\right)_{s}\right) .
$$

Then, as $J Z_{-}$is $\mathbb{F}$-predictable, it holds that

$$
\mathbb{E}\left(\int_{0}^{\infty} J_{s} Z_{s-} d\left(\Delta X_{\widetilde{R}} \mathbb{1}_{[\widetilde{R}, \infty[}\right)_{s}\right)=\mathbb{E}\left(\int_{0}^{\infty} J_{s} Z_{s-} d\left(\Delta X_{\widetilde{R}} \mathbb{1}_{[\widetilde{R}, \infty[}\right)_{s}^{p, \mathbb{F}}\right)
$$

and we obtain: 


$$
\begin{aligned}
\mathbb{E}\left(\left(H \cdot X^{\tau}\right)_{\infty}\right) & =\mathbb{E}\left(\int_{0}^{\tau} \frac{J_{s}}{\widetilde{Z}_{s}} \mathbb{1}_{\left\{\widetilde{Z}_{s}>0\right\}} d[X, m]_{s}\right)-\mathbb{E}\left(\int_{0}^{\infty} J_{s} Z_{s-} d\left(\Delta X_{\widetilde{R}} \mathbb{1}_{[\widetilde{R}, \infty[}\right)_{s}^{p, \mathbb{F}}\right) \\
& =\mathbb{E}\left(\int_{0}^{\tau} \frac{H_{s}}{\widetilde{Z}_{s}} \mathbb{1}_{\left\{\widetilde{Z}_{s}>0\right\}} d[X, m]_{s}\right)-\mathbb{E}\left(\int_{0}^{\tau} H_{s} d\left(\Delta X_{\widetilde{R}} \mathbb{1}_{[\widetilde{R}, \infty[}\right)_{s}^{p, \mathbb{F}}\right)
\end{aligned}
$$

where we have used the facts that $J$ is predictable and that $Z_{-}$is the predictable projection of $\mathbb{1}_{[0, \tau]}$. That ends the proof.

Remark 5. In [9, Paragraph 77, Chapter XX] an optional semimartingale decomposition is mentioned (without any proof) in the form: given an $\mathbb{F}$-local martingale $X$, the process

$$
\bar{X}_{t}:=X_{t}^{\tau}-\int_{0}^{t \wedge \tau} \frac{1}{\widetilde{Z}_{s}} d[X, m]_{s}
$$

is an $\mathbb{F}^{\tau}$-local martingale. This decomposition is valid for any $\mathbb{F}$-local martingale if and only if $\widetilde{R}=\infty \mathbb{P}$-a.s.. In particular, if all $\mathbb{F}$-martingales are continuous, then $\widetilde{R}=\infty \mathbb{P}$-a.s. and the above formula is valid. The condition $\widetilde{R}=\infty \mathbb{P}$-a.s. will play an important rôle in the study of stability of NUPBR condition.

Remark 6. The $\mathbb{F}^{\tau}$-local martingale $\bar{X}$ which appears in (9) can be expressed in terms of the $\mathbb{F}$-local martingale $N$ defined in (3). Indeed, from equalities $N=$ $N_{-}\left(\mathbb{1}_{\left\{Z_{-}>0\right\}} \frac{\widetilde{Z}}{Z_{-}}+\mathbb{1}_{\left\{Z_{-}=0\right\}}\right)$ and $N=1+N_{-} \frac{1}{Z_{-}} \mathbb{1}_{\left\{Z_{-}>0\right\}} \cdot m$ and the fact that $Z_{-}>0$ on $[0, \tau]$, it follows that

$$
\frac{1}{\widetilde{Z}} \mathbb{1}_{[0, \tau]} \cdot[X, m]=\frac{1}{N} \mathbb{1}_{[0, \tau]} \cdot[X, N] .
$$

We will now study some particular martingales which will be important for the construction, under adequate conditions, of deflators for price processes and we will give the relation of our construction with previous works, in particular [5, Proposition 3.6]. The next lemma defines an $\mathbb{F}^{\tau}$-local martingale $L^{\mathrm{pr}}$ which is the corner stone in the construction of the deflator ${ }^{2}$. Due to this lemma, we avoid the use of optional integrals done in equation (3.9) in [5, Proposition 3.6].

Lemma 2. (a) The $\mathbb{F}^{\tau}$-predictable process $\frac{1}{Z_{-}} \mathbb{1}_{[0, \tau]}$ is integrable with respect to $\bar{m}$, the $\mathbb{F}^{\tau}$-martingale part from the optional decomposition of m obtained in (9).

(b) Let

$$
L^{\mathrm{pr}}=\frac{1}{Z_{-}} \mathbb{1}_{[0, \tau]} \cdot \bar{m}
$$

Then

$$
L^{\mathrm{pr}}=\frac{Z_{-}^{2}}{Z_{-}^{2}+\Delta\langle m\rangle^{\mathbb{F}}} \frac{1}{\widetilde{Z}} \mathbb{1}_{[0, \tau]} \odot \widehat{m},
$$

where $\widehat{m}$ is the $\mathbb{F}^{\tau}$-local martingale part in the predictable decomposition of $m(8)$ and $\odot$ stands for the optional stochastic integral.

2 The upper script "pr" stands for progressive. 
Proof. In the proof, we set $L=L^{\mathrm{pr}}$ for simplicity.

(a) Being càglàd, the process $\frac{1}{Z_{-}} \mathbb{1}_{[0, \tau]}$ is locally bounded.

(b) The $\mathbb{F}^{\tau}$-continuous martingale part and the jump part of $\frac{1}{Z_{-}} \mathbb{1}_{[0, \tau]} \cdot \bar{m}$ are given by

$$
\begin{aligned}
\left(\frac{1}{Z_{-}} \mathbb{1}_{[0, \tau]} \cdot \bar{m}\right)^{c} & =\frac{1}{Z_{-}} \mathbb{1}_{[0, \tau]} \cdot\left(m^{c}-\frac{1}{Z_{-}} \mathbb{1}_{[0, \tau]} \cdot\left\langle m^{c}\right\rangle^{\mathbb{F}}\right) \\
\Delta\left(\frac{1}{Z_{-}} \mathbb{1}_{[0, \tau]} \cdot \bar{m}\right) & =\frac{\Delta m}{\widetilde{Z}} \mathbb{1}_{[0, \tau]}-p, \mathbb{F}\left(\mathbb{1}_{[\widetilde{R}]}\right) \mathbb{1}_{[0, \tau]},
\end{aligned}
$$

where $m^{c}$ is the $\mathbb{F}$-continuous martingale part of $m$. Let us now compute the $\mathbb{F}^{\tau}$ continuous martingale part and the jump part of $L$. By definition of the optional stochastic integral and Lemma 3.1 (b) in [5], we have:

$$
\begin{aligned}
L^{c} & =\frac{Z_{-}^{2}}{Z_{-}^{2}+\Delta\langle m\rangle^{\mathbb{F}}} p, \mathbb{F}^{\tau}\left(\frac{1}{\widetilde{Z}}\right) \mathbb{1}_{[0, \tau]} \cdot \widehat{m}^{c} \\
& =\frac{1}{Z_{-}} \mathbb{1}_{[0, \tau]} \cdot \widehat{m}^{c}-\frac{p, \mathbb{F}\left(\mathbb{1}_{\left\{\tilde{Z}=0<Z_{-}\right\}}\right)}{Z_{-}} \mathbb{1}_{[0, \tau]} \cdot \widehat{m}^{c} .
\end{aligned}
$$

As $\left\{\widetilde{Z}=0<Z_{-}\right\}$is a thin set, the set $\left\{p, \mathbb{F}\left(\mathbb{1}_{\left\{\widetilde{Z}=0<Z_{-}\right\}}\right) \neq 0\right\}$ is also thin, and from continuity of $\widehat{m}^{c}$, we conclude that

$$
L^{c}=\frac{1}{Z_{-}} \mathbb{1}_{[0, \tau]} \cdot \widehat{m}^{c}
$$

In the proof of Proposition 3.6 in [5], it is established that the jump process of $L$ is given by

$$
\Delta L=\frac{\Delta m}{\widetilde{Z}} \mathbb{1}_{[0, \tau]}-p, \mathbb{F}\left(\mathbb{1}_{\left\{\widetilde{Z}=0<Z_{-}\right\}}\right) \mathbb{1}_{[0, \tau]} .
$$

This completes the proof.

The link between the $\mathbb{F}^{\tau}$-local martingale $L^{\mathrm{pr}}$ and the $\mathbb{F}^{\tau}$-adapted process $\frac{1}{N^{\tau}}$, where $N$ is defined in (3), is made precise in the next lemma.

Proposition 6. Let $N$ be defined in (3).

(a) The process $\frac{1}{N^{\tau}}$ is an $\mathbb{F}^{\tau}$-supermartingale which can be written

$$
\frac{1}{N^{\tau}}=\mathscr{E}\left(-\left(L^{\mathrm{pr}}\right)^{\tau}-\left(\mathbb{1}_{[\widetilde{R}, \infty[}\right)_{\cdot \wedge \tau}^{p, \mathbb{F}}\right)
$$

(b) The process $\frac{1}{N^{\tau}}$ is an $\mathbb{F}^{\tau}$-local martingale if and only if $\widetilde{R}=\infty$. In that case $\frac{1}{N^{\tau}}=\mathscr{E}\left(-\left(L^{\mathrm{pr}}\right)^{\tau}\right)$.

Proof. (a) By Itô's formula and the obvious equality $d N=N_{-} \frac{1}{Z_{-}} \mathbb{1}_{\left\{Z_{-}>0\right\}} d m$ 


$$
\begin{aligned}
\frac{1}{N_{t}^{\tau}} & =1-\int_{0}^{t \wedge \tau} \frac{1}{N_{s-}^{2}} d N_{s}+\int_{0}^{t \wedge \tau} \frac{1}{N_{s-}^{3}} d\left\langle N^{c}\right\rangle_{s}+\sum_{s \leq t \wedge \tau}\left(\frac{1}{N_{s}}-\frac{1}{N_{s-}}+\frac{1}{N_{s-}^{2}} \Delta N_{s}\right) \\
& =1-\int_{0}^{t \wedge \tau} \frac{1}{N_{s-} Z_{s-}} d m_{s}+\int_{0}^{t \wedge \tau} \frac{1}{N_{s-} Z_{s-}^{2}} d\left\langle m^{c}\right\rangle_{s}+\sum_{s \leq t \wedge \tau} \frac{\left(\Delta m_{s}\right)^{2}}{N_{s-} Z_{s-} \widetilde{Z}_{s}},
\end{aligned}
$$

where we have used the fact that $\widetilde{Z}=Z_{-}+\Delta m$. We continue with

$$
\begin{aligned}
\frac{1}{N_{t}^{\tau}} & =1-\int_{0}^{t \wedge \tau} \frac{1}{N_{s-}} d\left(\frac{1}{Z_{-}} \cdot m-\frac{1}{Z_{-}^{2}} \cdot\left\langle m^{c}\right\rangle-\sum \frac{(\Delta m)^{2}}{Z_{-} \widetilde{Z}}\right)_{s} \\
& =1-\int_{0}^{t \wedge \tau} \frac{1}{N_{s-}} d\left(\frac{1}{Z_{-}} \cdot \bar{m}+\left(\mathbb{1}_{[\widetilde{R}, \infty[}\right)^{p, \mathbb{F}}\right)_{s}
\end{aligned}
$$

where the second equality comes from Theorem 3 applied to the $\mathbb{F}$-martingale $m$. Finally we conclude that

$$
\frac{1}{N^{\tau}}=\mathscr{E}\left(-\frac{1}{Z_{-}} \mathbb{1}_{[0, \tau]} \cdot \bar{m}-\left(\mathbb{1}_{[\widetilde{R}, \infty[}\right)_{. \wedge \tau}^{p, \mathbb{F}}\right)
$$

(b) From the previous equality, we see that the process $\frac{1}{N^{\tau}}$ is an $\mathbb{F}^{\tau}$-local martingale if and only if $\left(\mathbb{1}_{[\widetilde{R}, \infty[}\right)_{\cdot \wedge \tau}^{p, \mathbb{F}}=0$. The last equality is equivalent to

$$
0=\mathbb{E}\left(\left(\mathbb{1}_{[\widetilde{R}, \infty[}\right)_{\tau}^{p, \mathbb{F}}\right)=\mathbb{E}\left(\int_{0}^{\infty} Z_{s-} d\left(\mathbb{1}_{[\widetilde{R}, \infty[}\right)_{s}^{p, \mathbb{F}}\right)=\mathbb{E}\left(Z_{\widetilde{R}-} \mathbb{1}_{\{\widetilde{R}<\infty\}}\right),
$$

which in turn is equivalent to $\widetilde{R}=\infty, \mathbb{P}$-a.s. since $Z_{\widetilde{R}-}>0$ on $\{\widetilde{R}<\infty\}$.

\subsection{Deflators for progressive enlargement up to $\tau$}

In this section, we give alternative proofs, based on the optional semimartingale decomposition, to results in [1] and to Theorem 2.23 and Corollary 2.18 (c) from [5] (or their general versions in [4]). In Proposition 7 (a), we determine an $\mathbb{F}^{\tau}$-local martingale deflator for a large class of $\mathbb{F}$-local martingales. In Proposition 7 (b), an $\mathbb{F}^{\tau}$-supermartingale deflator for $\mathbb{F}$-local martingales is studied.

We introduce an $\mathbb{F}^{\tau}$-predictable process $V^{\text {pr }}$ which is crucial for proofs therein (also used in [5]). Denoting by $\widetilde{R}^{a}$ the accessible part of the $\mathbb{F}$-stopping time $\widetilde{R}$, we set

$$
V_{t}^{\mathrm{pr}}:=\left(\mathbb{1}_{\left[\widetilde{R}^{a}, \infty[\right.}\right)_{t \wedge \tau}^{p, \mathbb{F}}
$$

Using the process $V^{\mathrm{pr}}$ we study, in the next proposition, a particular $\mathbb{F}^{\tau}$-supermartingale which will play the rôle of a deflator for some $\mathbb{F}$-local martingales.

Proposition 7. Assume that $X$ is an $\mathbb{F}$-local martingale such that 


$$
\Delta X_{\widetilde{R}}=0 \text { on }\{\widetilde{R}<\infty\} .
$$

(a) If $X$ is quasi-left continuous, then $Y^{\mathrm{pr}}:=\mathscr{E}\left(-L^{\mathrm{pr}}\right)$ is an $\mathbb{F}^{\tau}$-local martingale deflator for $X^{\tau}$.

(b) The process $\widetilde{Y}^{\mathrm{pr}}:=\mathscr{E}\left(-L^{\mathrm{pr}}-V^{\mathrm{pr}}\right)$ is an $\mathbb{F}^{\tau}$-supermartingale deflator for $X^{\tau}$.

Proof. (a) In the proof, we set $Y=Y^{\mathrm{pr}}$ and $L=L^{\mathrm{pr}}$ for simplicity. Using integration by parts and the optional decomposition (9) given in Theorem 3 for $X$ and then for $m$, we obtain:

$$
\begin{aligned}
& Y X^{\tau}=X_{-}^{\tau} \cdot Y+Y_{-} \cdot X^{\tau}+\left[Y, X^{\tau}\right] \\
& =X_{-}^{\tau} \cdot Y+Y_{-} \cdot \bar{X}+Y_{-} \frac{1}{\widetilde{Z}} \mathbb{1}_{[0, \tau]} \cdot[m, X]-Y_{-} \mathbb{1}_{[0, \tau]} \cdot\left(\Delta X_{\widetilde{R}} \mathbb{1}_{[\widetilde{R}, \infty[}\right)^{p, \mathbb{F}}-Y_{-} \mathbb{1}_{[0, \tau]} \cdot[L, X] \\
& =X_{-}^{\tau} \cdot Y+Y_{-} \cdot \bar{X}+Y_{-} \frac{1}{\widetilde{Z}} \mathbb{1}_{[0, \tau]} \cdot[\bar{m}, X]+Y_{-} \frac{1}{\widetilde{Z}^{2}} \mathbb{1}_{[0, \tau]} \cdot[[m], X] \\
& -Y_{-} \frac{1}{\widetilde{Z}} \mathbb{1}_{[0, \tau]} \cdot\left[\left(\Delta m_{\widetilde{R}} \mathbb{1}_{[\widetilde{R}, \infty[}\right)^{p, \mathbb{F}}, X\right]-Y_{-} \mathbb{1}_{[0, \tau]} \cdot\left(\Delta X_{\widetilde{R}} \mathbb{1}_{[\widetilde{R}, \infty[}\right)^{p, \mathbb{F}}-\frac{Y_{-}}{Z_{-}} \mathbb{1}_{[0, \tau]} \cdot[\bar{m}, X] \\
& =: I_{1}+I_{2}+I_{3}+I_{4}+I_{5}+I_{6}+I_{7} \text {. }
\end{aligned}
$$

In a first step, we study the sum of third and seventh term of the last expression

$$
\begin{aligned}
I_{3}+I_{7} & =Y_{-}\left(\frac{1}{\widetilde{Z}}-\frac{1}{Z_{-}}\right) \mathbb{1}_{[0, \tau]} \cdot[\bar{m}, X]=-Y_{-} \frac{\Delta m}{\widetilde{Z} Z_{-}} \mathbb{1}_{[0, \tau]} \cdot[\bar{m}, X] \\
& =-\sum Y_{-} \frac{\Delta m}{\widetilde{Z} Z_{-}} \mathbb{1}_{[0, \tau]} \Delta \bar{m} \Delta X,
\end{aligned}
$$

where the third equality comes from the fact that $\{\Delta m \neq 0\}$ is a thin set. We add the term $I_{4}$ to the previous two

$$
\begin{aligned}
I_{4}+\left(I_{3}+I_{7}\right) & :=\sum Y_{-} \frac{1}{\widetilde{Z}^{2}} \mathbb{1}_{[0, \tau]}(\Delta m)^{2} \Delta X-\sum Y_{-} \frac{\Delta m}{\widetilde{Z} Z_{-}} \mathbb{1}_{[0, \tau]} \Delta \bar{m} \Delta X \\
& =-\sum Y_{-} \frac{\Delta m}{\widetilde{Z}} \Delta X \mathbb{1}_{[0, \tau]}\left(\frac{1}{Z_{-}} \Delta \bar{m}-\frac{1}{\widetilde{Z}} \Delta m\right) \\
& =\sum Y_{-} \frac{\Delta m}{\widetilde{Z}} \Delta X \mathbb{1}_{[0, \tau]} p, \mathbb{F}\left(\mathbb{1}_{[\widetilde{R}]}\right),
\end{aligned}
$$

where the last equality comes from (10). Note that, by Yoeurp's lemma (which states that, for a predictable bounded variation process $V$ and a semimartingale $Y,[V, Y]=$ $\Delta V . X$, see, e.g., [18, Proposition 9.3.7.1]), properties of dual predictable projection, and the fact that $p(\Delta V)=\Delta\left(V^{p}\right)$, the fifth term in the expression for $Y X^{\tau}$ is equal to

$$
\begin{aligned}
I_{5} & =-Y_{-} \frac{1}{\widetilde{Z}} \mathbb{1}_{[0, \tau]} \cdot\left[\left(\Delta m_{\widetilde{R}} \mathbb{1}_{[\widetilde{R}, \infty]}\right)^{p, \mathbb{F}}, X\right]=-Y_{-} \frac{1}{\widetilde{Z}} \mathbb{1}_{[0, \tau]}^{p, \mathbb{F}}\left(\Delta m_{\widetilde{R}} \mathbb{1}_{[\widetilde{R}]}\right) \cdot X \\
& =\sum Y_{-} \frac{Z_{-}}{\widetilde{Z}} \mathbb{1}_{[0, \tau]}^{p, \mathbb{F}}\left(\mathbb{1}_{[\widetilde{R}]}\right) \Delta X,
\end{aligned}
$$


where the last equality is due to $\Delta m_{\widetilde{R}}=-Z_{\widetilde{R}-}$ and the fact that the process ${ }^{p, \mathbb{F}}\left(\mathbb{1}_{[\widetilde{R}]}\right)$ is thin.

Finally, using the fact that $Z_{-}+\Delta m=\widetilde{Z}$, we get

$$
\begin{aligned}
I_{5}+\left(I_{4}+I_{3}+I_{7}\right) & =\sum Y_{-} \frac{Z_{-}}{\widetilde{Z}} \mathbb{1}_{[0, \tau]} p, \mathbb{F}\left(\mathbb{1}_{[\widetilde{R}]}\right) \Delta X+\sum Y_{-} \frac{\Delta m}{\widetilde{Z}} \Delta X \mathbb{1}_{[0, \tau]} p, \mathbb{F}\left(\mathbb{1}_{[\widetilde{R}]}\right) \\
& =\sum Y_{-} \mathbb{1}_{[0, \tau]} p, \mathbb{F}\left(\mathbb{1}_{[\widetilde{R}]}\right) \Delta X .
\end{aligned}
$$

Summing up we have that

$$
Y X^{\tau}=X_{-}^{\tau} \cdot Y+Y_{-} \cdot \bar{X}+\sum Y_{-} \mathbb{1}_{[0, \tau]}^{p, \mathbb{F}}\left(\mathbb{1}_{[\widetilde{R}]}\right) \Delta X-Y_{-} \mathbb{1}_{[0, \tau]} \cdot\left(\Delta X_{\widetilde{R}} \mathbb{1}_{[\widetilde{R}, \infty[}\right)^{p, \mathbb{F}} .
$$

If $X$ is an $\mathbb{F}$-quasi-left continuous local martingale, using the predictability of $p, \mathbb{F}\left(\mathbb{1}_{[\widetilde{R}]}\right)$ and $\Delta X_{\widetilde{R}}=0$ on $\{\widetilde{R}<\infty\}$, then

$$
Y X^{\tau}=X_{-}^{\tau} \cdot Y+Y_{-} \cdot \bar{X}
$$

which implies that $Y X^{\tau}$ is a local martingale, hence $Y$ is an $\mathbb{F}^{\tau}$-local martingale deflator for $X^{\tau}$.

(b) In the proof, we set $\widetilde{Y}=\widetilde{Y}^{\mathrm{pr}}, L=L^{\mathrm{pr}}$ and $V=V^{\mathrm{pr}}$ for simplicity. Let $H$ be an $\mathbb{F}^{\tau}$-predictable process such that $H \cdot X \geq-1$. By integration by parts, we get

$$
\left(1+H \cdot X^{\tau}\right) \widetilde{Y}=\left(1+H \cdot X^{\tau}\right)_{-} \cdot \widetilde{Y}+H \widetilde{Y}_{-} \cdot X^{\tau}-H \widetilde{Y}_{-} \cdot\left[X^{\tau}, L\right]-H \widetilde{Y}_{-} \cdot\left[X^{\tau}, V\right] .
$$

Note that

$$
H \widetilde{Y}_{-} \cdot\left[X^{\tau}, V\right]=\sum H \widetilde{Y}_{-} \mathbb{1}_{[0, \tau]} p, \mathbb{F}\left(\mathbb{1}_{[\widetilde{R}]}\right) \Delta X .
$$

Then, using the same arguments as in the proof of (a), we get

$$
\left(1+H \cdot X^{\tau}\right) \widetilde{Y}=\left(1+H \cdot X^{\tau}\right)_{-} \cdot \widetilde{Y}+H \widetilde{Y}_{-} \cdot \bar{X}-H \widetilde{Y}_{-} \mathbb{1}_{[0, \tau]} \cdot\left(\Delta X_{\widetilde{R}} \mathbb{1}_{[\widetilde{R}, \infty[}\right)^{p, \mathbb{F}} .
$$

In particular, if $\Delta X_{\widetilde{R}}=0$ on $\{\widetilde{R}<\infty\}$, then $\widetilde{Y}$ is an $\mathbb{F}^{\tau}$-supermartingale deflator for $X^{\tau}$ and $X^{\tau}$ satisfies $\operatorname{NUPBR}\left(\mathbb{F}^{\tau}\right)$. This ends the proof of the proposition.

Proposition 8. Let $X$ be a process such that $\Delta X_{\widetilde{R}}=0$ on $\{\widetilde{R}<\infty\}$ and admitting an $\mathbb{F}$-local martingale deflator. Then $X^{\tau}$ admits an $\mathbb{F}^{\tau}$-local martingale deflator.

Proof. There exist a real-valued $\mathbb{F}$-predictable process $\phi$ and a positive $\mathbb{F}$-local martingale $K$ such that

$$
0<\phi \leq 1 \quad \text { and } \quad K(\phi \cdot X) \quad \text { is an } \mathbb{F} \text {-local martingale. }
$$

Then there exists a sequence of $\mathbb{F}$-stopping times $\left(v_{n}\right)_{n}$ that increases to infinity such that the stopped process $K^{v_{n}}$ is an $\mathbb{F}$-martingale. Put $\mathbb{Q}_{n}:=K_{v_{n}} \mathbb{P} \sim \mathbb{P}$. Then, by applying Proposition 7 to $(\phi \cdot X)^{v_{n}}$ under $\mathbb{Q}_{n}$, we conclude that $(\phi \cdot X)^{v_{n} \wedge \tau}$ satisfies $\operatorname{NUPBR}\left(\mathbb{F}^{\tau}\right)$ under $\mathbb{Q}_{n}$. Thanks to Proposition 5, NUPBR $\left(\mathbb{F}^{\tau}\right)$ under $\mathbb{P}$ of $X^{\tau}$ follows immediately. 
The next result provides explicit $\mathbb{F}^{\tau}$-local martingale deflators for $\mathbb{F}$-local martingales. The proof differs from the one of Theorem [5, Theorem 2.23] and is based on the optional semimartingale decomposition and direct computations.

Theorem 4. The following conditions are equivalent.

(a) The thin set $\left\{\widetilde{Z}=0<Z_{-}\right\}$is evanescent.

(b) The $\mathbb{F}$-stopping time $\widetilde{R}$ is infinite $(\widetilde{R}=\infty)$.

(c) For any $\mathbb{F}$-local martingale $X$, the process $X^{\tau}$ admits $Y^{\mathrm{pr}}$ as $\mathbb{F}^{\tau}$-local martingale deflator, hence, satisfies $\operatorname{NUPBR}\left(\mathbb{F}^{\tau}\right)$.

(d) For any (bounded) process $X$ satisfying $N U P B R(\mathbb{F})$, the process $X^{\tau}$ satisfies $\operatorname{NUPBR}\left(\mathbb{F}^{\tau}\right)$.

Proof. The equivalence between (a) and (b) is obvious from definition of $\widetilde{R}$.

The implication $(b) \Rightarrow(c)$ follows from Proposition 7 . To prove $(c) \Rightarrow(b)$ (and $(d) \Rightarrow(b)$ ), we consider the $\mathbb{F}$-martingale

$$
X=\mathbb{1}_{[\widetilde{R}, \infty[}-\left(\mathbb{1}_{[\widetilde{R}, \infty[}\right)^{p, \mathbb{F}}
$$

Note that $\mathbb{P}(\tau=\widetilde{R})=\mathbb{E}\left(\Delta A_{\widetilde{R}}^{o}\right)=\mathbb{E}\left(\widetilde{Z}_{\widetilde{R}}-Z_{\widetilde{R}}\right)=0$ (since $0=\widetilde{Z}_{\widetilde{R}} \geq Z_{\widetilde{R}} \geq 0$ ). This implies that $\tau<\widetilde{R}$ and

$$
X^{\tau}=-\left(\mathbb{1}_{[\widetilde{R}, \infty[}\right)_{\cdot \wedge \tau}^{p, \mathbb{F}}
$$

is a predictable decreasing process. Thus, from [5, lemma 2.6], $X^{\tau} \operatorname{satisfies} \operatorname{NUPBR}\left(\mathbb{F}^{\tau}\right)$ if and only if it is a null process. Then, we conclude that $\widetilde{R}$ is infinite using the same argument as in the proof of Lemma 6 (b). The implication (c) $\Rightarrow$ (d) follows from Proposition 8.

\section{Initial Enlargement under Jacod's Hypothesis}

In this section, we study initial enlargement of filtration and NUPBR condition under absolute continuity Jacod's hypothesis. We extend some results of Amendinger [6] that require both equivalence Jacods hypothesis and Theorem 1.

\subsection{Optional semimartingale decomposition for initial enlargement}

In this subsection, we develop our $\mathbb{F}^{\sigma(\xi)}$-optional semimartingale decomposition of parametrized $\mathbb{F}$-local martingales. We first decompose the $\mathbb{F}$-stopping time $R^{u}$, introduced in (4), as $R^{u}=\widetilde{R}^{u} \wedge \bar{R}^{u}$ with

$$
\left.\widetilde{R}^{u}=R_{\left\{q_{R_{-}}^{u}>0\right\}}^{u} \quad \text { and } \quad \bar{R}^{u}=R_{\left\{q_{R^{u}-}^{u}\right.}^{u}=0\right\} .
$$


Clearly $\bar{R}^{u}$ is an $\mathbb{F}$-predictable stopping time and $\left\{R^{u}=\infty\right\} \subset\left\{\bar{R}^{u}=\infty\right\}$ so

$$
\left.\left(\mathbb{1}_{\left[R^{u}, \infty[\right.}\right)^{p, \mathbb{F}}\right|_{u=\xi}=\left.\left(\mathbb{1}_{\left[\widetilde{R}^{u}, \infty[\right.}\right)^{p, \mathbb{F}}\right|_{u=\xi}
$$

In the following lemma, we express the $\mathbb{F}^{\sigma(\xi)}$-dual predictable projection in terms of the $\mathbb{F}$-dual predictable projection. This is a result for initial enlargement case similar to the one given in [5, Lemma 3.1 (a) and Lemma 3.2] for progressive enlargement case.

Lemma 3. Let $\left(V^{u}, u \in \mathbb{R}\right)$ be a parametrized $\mathbb{F}$-adapted càdlàg process with locally integrable variation $\left(V \in \mathscr{A}_{\text {loc }}(\mathbb{F})\right)$. Then the following properties hold:

(a) The $\mathbb{F}^{\sigma(\xi)}$-dual predictable projection of $V^{\xi}$ is

$$
\left(V^{\xi}\right)^{p, \mathbb{F}^{\sigma(\xi)}}=\left.\frac{1}{q_{-}^{\xi}} \cdot\left(q^{u} \cdot V^{u}\right)^{p, \mathbb{F}}\right|_{u=\xi}
$$

(b) If $\left(V^{u}, u \in \mathbb{R}\right)$ belongs to $\mathscr{A}_{\text {loc }}^{+}(\mathbb{F})$ (respectively $V \in \mathscr{A}^{+}(\mathbb{F})$ ), then the process $\left(U^{u}, u \in \mathbb{R}\right)$ with

$$
U^{u}:=\frac{1}{q^{\xi}} \cdot V^{u},
$$

belongs to $\mathscr{A}_{\text {loc }}^{+}\left(\mathbb{F}^{\sigma(\xi)}\right)$ (respectively to $\mathscr{A}^{+}\left(\mathbb{F}^{\sigma(\xi)}\right)$ ).

(c) If $\left(V^{u}, u \in \mathbb{R}\right)$ belongs to $\mathscr{A}_{\text {loc }}(\mathbb{F})$, the process $\left(U^{u}=\frac{1}{q^{\xi}} \cdot V^{u}, u \in \mathbb{R}\right)$ is well defined, its variation is $\mathbb{F}^{\sigma(\xi)}$-locally integrable, and the $\mathbb{F}^{\sigma(\xi)}$-dual predictable projection of $U^{\xi}$ is given by

$$
\left(U^{\xi}\right)^{p, \mathbb{F}^{\sigma(\xi)}}=\left.\frac{1}{q_{-}^{\xi}} \cdot\left(\mathbb{1}_{\left\{q^{u}>0\right\}} \cdot V^{u}\right)^{p, \mathbb{F}}\right|_{u=\xi} .
$$

Proof. (a) We apply the predictable semimartingale decomposition given in Proposition 4 to the parametrized $\mathbb{F}$-local martingale $\left(X^{u}, u \in \mathbb{R}\right)=\left(V^{u}-\left(V^{u}\right)^{p, \mathbb{F}}, u \in \mathbb{R}\right)$, obtaining

$$
\begin{aligned}
V^{\xi} & =\widehat{X}^{\xi}+\left.\left(V^{u}\right)^{p, \mathbb{F}}\right|_{u=\xi}+\left.\frac{1}{q_{-}^{\xi}} \cdot\left\langle V^{u}, q^{u}\right\rangle^{\mathbb{F}}\right|_{u=\xi} \\
& =\widehat{X}^{\xi}+\left.\left(\mathbb{1}_{\left\{q^{u}>0\right\}} \cdot V^{u}\right)^{p, \mathbb{F}}\right|_{u=\xi}+\left.\left(\frac{\Delta q^{u}}{q_{-}^{u}} \mathbb{1}_{\left\{q^{u}>0\right\}} \cdot V^{u}\right)^{p, \mathbb{F}}\right|_{u=\xi} \\
& =\widehat{X}^{\xi}+\left.\frac{1}{q_{-}^{\xi}} \cdot\left(q^{u} \cdot V^{u}\right)^{p, \mathbb{F}}\right|_{u=\xi},
\end{aligned}
$$

which proves assertion (a).

(b) Suppose that $\left(V^{u}, u \in \mathbb{R}\right) \in \mathscr{A}_{l o c}^{+}(\mathbb{F})$. For fixed $u$, let $\left(\vartheta_{n}\right)_{n \geq 1}$ be a sequence of $\mathbb{F}$ stopping times that increases to infinity such that $\mathbb{E}\left(V_{\vartheta_{n}}^{u}\right)<\infty$. Then, $\mathbb{E}\left(U_{\vartheta_{n}}^{u}\right)<\infty$ since 


$$
\mathbb{E}\left(U_{\vartheta_{n}}^{u}\right)=\mathbb{E}\left(\int_{0}^{\vartheta_{n}} \frac{1}{q_{t}^{\xi}} d V_{t}^{u}\right)=\mathbb{E}\left(\int_{0}^{\vartheta_{n}} \int_{\mathbb{R}} \mathbb{1}_{\left\{q_{t}^{y}>0\right\}} \eta(d y) d V_{t}^{u}\right) \leq \mathbb{E}\left(V_{\vartheta_{n}}^{u}\right)<\infty,
$$

where the last equality comes from (6) applied to $\frac{1}{q_{t}^{u}} \mathbb{1}_{\left\{q_{t}^{u}>0\right\}}$.

(c) Suppose that $\left(V^{u}, u \in \mathbb{R}\right) \in \mathscr{A}_{\text {loc }}(\mathbb{F})$, and denote by $W:=V^{+}+V^{-}$its variation. Then $\left(W^{u}, u \in \mathbb{R}\right) \in \mathscr{A}_{\text {loc }}^{+}(\mathbb{F})$, and a direct application of (b) implies that

$$
\left(\frac{1}{q^{\xi}} \cdot W^{u}, u \in \mathbb{R}\right) \in \mathscr{A}_{l o c}^{+}\left(\mathbb{F}^{\tau}\right) .
$$

As a result, we deduce that $U$ given by (13) for the case of $V=V^{+}-V^{-}$is well defined and has variation equal to $\frac{1}{q^{\xi}}$. W which is $\mathbb{F}^{\sigma(\xi)}$-locally integrable. For each $n \geq 1$, let us consider the parametrized process $\left(U_{n}^{u}, u \in \mathbb{R}\right)$ with

$$
U_{n}^{u}:=\frac{1}{q^{u}} \mathbb{1}_{\left\{q^{u} \geq \frac{1}{n}\right\}} \cdot V^{u} .
$$

Due to (12), we derive

$$
\left(U_{n}^{\xi}\right)^{p, \mathbb{F}^{\sigma(\xi)}}=\left.\frac{1}{q_{-}^{\xi}} \cdot\left(\mathbb{1}_{\left\{q^{u} \geq 1 / n\right\}} \cdot V^{u}\right)^{p, \mathbb{F}}\right|_{u=\xi} .
$$

Hence, since $\left(U^{\xi}\right)^{p, \mathbb{F}^{\sigma(\xi)}}=\lim _{n \rightarrow \infty}\left(U_{n}^{\xi}\right)^{p, \mathbb{F}^{\sigma(\xi)}}$ by taking the limit in the above equality, we get

$$
\left(U^{\xi}\right)^{p, \mathbb{F}^{\sigma(\xi)}}=\left.\frac{1}{q_{-}^{\xi}} \cdot\left(\mathbb{1}_{\left\{q^{u}>0\right\}} \cdot V^{u}\right)^{p, \mathbb{F}}\right|_{u=\xi} \cdot
$$

This ends the proof.

Remark 7. The above lemma allows us to make precise the link between predictable brackets in $\mathbb{F}$ and in $\mathbb{G}$. Indeed, for two $\mathbb{F}$ martingales $X$ and $Y$

$$
\begin{aligned}
\langle X, Y\rangle^{\mathbb{F}^{\sigma(\xi)}} & =([X, Y])^{p, \mathbb{F}^{\sigma(\xi)}}=\left.\frac{1}{q_{-}^{\xi}} \cdot\left(q^{u} \cdot[X, Y]\right)^{p, \mathbb{F}}\right|_{u=\xi} \\
& =\left.\frac{1}{q_{-}^{\xi}} \cdot\left(q_{-}^{u} \cdot[X, Y]\right)^{p, \mathbb{F}}\right|_{u=\xi}+\left.\frac{1}{q_{-}^{\xi}} \cdot\left(\Delta q^{u} \cdot[X, Y]\right)^{p, \mathbb{F}}\right|_{u=\xi} \\
& =\langle X, Y\rangle^{\mathbb{F}}+\left.\left(\sum \frac{\Delta q^{u}}{q_{-}^{u}} \Delta X \Delta Y\right)^{p, \mathbb{F}}\right|_{u=\xi} .
\end{aligned}
$$

We are now ready to state, in the next theorem, the main result of this section with a proof based on Lemma 3 .

Theorem 5. Let $\left(X^{u}, u \in \mathbb{R}\right)$ be a parametrized $\mathbb{F}$-local martingale. Then, 


$$
\bar{X}_{t}^{\xi}:=X_{t}^{\xi}-\int_{0}^{t} \frac{1}{q_{s}^{\xi}} d\left[X^{\xi}, q^{\xi}\right]_{s}+\left.\left(\Delta X_{\widetilde{R}^{u}}^{u} \mathbb{1}_{\left[\widetilde{R}^{u}, \infty[\right.}\right)_{t}^{p, \mathbb{F}}\right|_{u=\xi}
$$

is an $\mathbb{F}^{\sigma(\xi)}$-local martingale. Here, $\widetilde{R}^{u}$ is defined in (11).

Proof. From the predictable decomposition given in Proposition $4, X^{\xi}$ can be written as

$$
X^{\xi}=\widehat{X}^{\xi}+\left.\frac{1}{q_{-}^{\xi}} \cdot\left(\mathbb{1}_{\left\{q^{u}>0\right\}} \cdot\left[X^{u}, q^{u}\right]\right)^{p, \mathbb{F}}\right|_{u=\xi}+\left.\frac{1}{q_{-}^{\xi}} \cdot\left(\mathbb{1}_{\left\{q^{u}=0\right\}} \cdot\left[X^{u}, q^{u}\right]\right)^{p, \mathbb{F}}\right|_{u=\xi}
$$

Using Lemma 3 (c) and the fact that $\Delta q_{\widetilde{R}^{u}}^{u}=-q_{\widetilde{R}^{u}-}^{u}$

$$
\begin{aligned}
X^{\xi} & =\widehat{X}^{\xi}+\left(\frac{1}{q^{\xi}} \cdot\left[X^{\xi}, q^{\xi}\right]\right)^{p, \mathbb{F}^{\sigma(\xi)}}+\left.\frac{1}{q_{-}^{\xi}} \cdot\left(\Delta X_{\widetilde{R}^{u}}^{u} \Delta q_{\widetilde{R}^{u}}^{u} \mathbb{1}_{\left[\widetilde{R}^{u}, \infty[\right.}\right)^{p, \mathbb{F}}\right|_{u=\xi} \\
& =\bar{X}^{\xi}+\frac{1}{q^{\xi}} \cdot\left[X^{\xi}, q^{\xi}\right]-\left.\left(\Delta X_{\widetilde{R}^{u}}^{u} \mathbb{1}_{\left[\widetilde{R}^{u}, \infty[\right.}\right)^{p, \mathbb{F}}\right|_{u=\xi}
\end{aligned}
$$

where

$$
\bar{X}^{\xi}:=\widehat{X}^{\xi}-\frac{1}{q^{\xi}} \cdot\left[X^{\xi}, q^{\xi}\right]+\left(\frac{1}{q^{\xi}} \cdot\left[X^{\xi}, q^{\xi}\right]\right)^{p, \mathbb{F}^{\sigma(\xi)}}
$$

is proved to be an $\mathbb{F}^{\sigma(\xi)}$-local martingale.

In [6], the process $\frac{1}{q^{\xi}}$ was studied in the case of a random variable $\xi$ satisfying equivalence Jacod's hypothesis, and was proved to be an $\mathbb{F}^{\sigma(\xi)}$-local martingale. Here we work under a weaker assumption, and we show that the martingale property established in [6] fails in the general case.

In the next two lemmas, we study the properties of the process $q^{\xi}$. In Lemma 4 we define particular $\mathbb{F}^{\sigma(\xi)}$-local martingales based on $q^{\xi}$. Then, in Lemma 5, we focus on the process $\frac{1}{q^{\xi}}$, which is proved to be an $\mathbb{F}^{\sigma(\xi)}$-supermartingale, and we give its semimartingale decomposition. We give a necessary and sufficient condition such that $\frac{1}{q^{\xi}}$ is an $\mathbb{F}^{\sigma(\xi)}$-local martingale.

Lemma 4. Let $\bar{q}^{\xi}$ be the $\mathbb{F}^{\sigma(\xi)}$-local martingale part of $q^{\xi}$ given by (14), i.e.,

$$
\bar{q}^{\xi}:=q^{\xi}-\frac{1}{q^{\xi}} \cdot\left[q^{\xi}\right]-\left.q_{-}^{\xi} \cdot\left(\mathbb{1}_{\left[\widetilde{R}^{u}, \infty[\right.}\right)^{p, \mathbb{F}}\right|_{u=\xi} .
$$

Then, the $\mathbb{F}^{\sigma(\xi)}$-predictable process $\frac{1}{q_{-}^{\xi}}$ is integrable with respect to $\bar{q}^{\xi}$ and the $\mathbb{F}^{\sigma(\xi)}$-local martingale

$$
L^{\mathrm{i}}:=\frac{1}{q_{-}^{\xi}} \cdot \bar{q}^{\xi}
$$


is such that $1-\Delta L^{\mathrm{i}}>0 .^{3}$

Proof. The process $\frac{1}{q_{-}^{\xi}}$ is càglàd so it is locally bounded.

The definition of $\bar{q}^{\xi}$ implies

$$
\begin{aligned}
1-\Delta L^{\mathrm{i}} & =1-\frac{1}{q_{-}^{\xi}}\left(\Delta q^{\xi}-\frac{1}{q^{\xi}}\left(\Delta q^{\xi}\right)^{2}\right)+\left.\Delta\left(\mathbb{1}_{\widetilde{R}^{u}, \infty}\right)^{p, \mathbb{F}}\right|_{u=\xi} \\
& =1-\frac{\Delta q^{\xi}}{q^{\xi}}+p,\left.\mathbb{F}\left(\mathbb{1}_{\left[\widetilde{R}^{u}\right]}\right)\right|_{u=\xi} \\
& =\frac{q_{-}^{\xi}}{q^{\xi}}+\left.{ }^{p, \mathbb{F}}\left(\mathbb{1}_{\left[\widetilde{R}^{u}\right]}\right)\right|_{u=\xi}>0,
\end{aligned}
$$

which completes the proof.

Under equivalent Jacod's hypothesis, as stated in Theorem 1, the process $\frac{1}{q^{\xi}}$ is true $\mathbb{F}^{\sigma(\xi)}$-martingale and provides an interesting change of probability.

Lemma 5. (a) The process $\frac{1}{q^{\xi}}$ is an $\mathbb{F}^{\sigma(\xi)}$-supermartingale with Doob-Meyer decomposition

$$
\frac{1}{q^{\xi}}=1-\frac{1}{\left(q^{\xi}\right)_{-}^{2}} \cdot \bar{q}^{\xi}-\left.\frac{1}{q_{-}^{\xi}} \cdot\left(\mathbb{1}_{\left[\widetilde{R}^{u}, \infty[\right.}\right)^{p, \mathbb{F}}\right|_{u=\xi} .
$$

Equivalently, it can be written as a stochastic exponential of the form

$$
\frac{1}{q^{\xi}}=\mathscr{E}\left(-L^{\mathrm{i}}-\left.\left(\mathbb{1}_{\left[\widetilde{R}^{u}, \infty[\right.}\right)^{p, \mathbb{F}}\right|_{u=\xi}\right) .
$$

(b) The process $\frac{1}{a^{\xi}}$ is an $\mathbb{F}^{\sigma(\xi)}$-local martingale if and only if $\widetilde{R}^{u}=\infty \mathbb{P} \otimes \eta$-a.s. Then $\frac{1}{q^{\xi}}=\mathscr{E}\left(-L^{\mathrm{i}}\right)$.

(c) In particular, the process $\frac{1}{q^{\xi}}$ is a true $\mathbb{F}^{\sigma(\xi)}$-martingale if and only if $R^{u}=\infty$ $\mathbb{P} \otimes \eta$-a.s. (i.e., under equivalent Jacod's hypothesis).

Proof. (a) $\left(q^{u}, u \in \mathbb{R}\right)$ is a parametrized $\mathbb{F}$-martingale, then by Proposition $4, q^{\xi}$ is an $\mathbb{F}^{\sigma(\xi)}$-semimartingale. By (7), $q^{\xi}$ is strictly positive. Then, $\frac{1}{q^{\xi}}$ is an $\mathbb{F}^{\sigma(\xi)}$ semimartingale, and by definition of the bracket, as

$$
\frac{1}{q^{\xi}}=1-\frac{1}{\left(q^{\xi}\right)_{-}^{2}} \cdot q^{\xi}+\frac{1}{\left(q^{\xi}\right)_{-}^{2} q^{\xi}} \cdot\left[q^{\xi}\right] .
$$

Applying (14), we finally get that

$$
\frac{1}{q^{\xi}}=1-\frac{1}{\left(q^{\xi}\right)_{-}^{2}} \cdot \bar{q}^{\xi}-\left.\frac{1}{q_{-}^{\xi}} \cdot\left(\mathbb{1}_{\left[\widetilde{R}^{u}, \infty[\right.}\right)^{p, \mathbb{F}}\right|_{u=\xi} \cdot
$$

${ }^{3}$ The upper script "i" stands for initial. 
The exponential form immediately follows.

(b) Since $\left.\frac{1}{q_{-}^{\xi}} \cdot\left(\mathbb{1}_{\left[\tilde{R}^{u}, \infty\right]}\right)^{p, \mathbb{F}}\right|_{u=\xi}$ is an $\mathbb{F}^{\sigma(\xi)}$-predictable increasing process, the process $\frac{1}{q^{\xi}}$ is an $\mathbb{F}^{\sigma(\xi)}$-local martingale if and only if $\left.\left(\mathbb{1}_{\left[\widetilde{R}^{u}, \infty[\right.}\right)^{p, \mathbb{F}}\right|_{u=\xi}=0$. The last condition is equivalent to have that, for each $t$

$$
\begin{aligned}
0 & =\mathbb{E}\left(\left.\left(\mathbb{1}_{\left[\widetilde{R}^{u}, \infty[\right.}\right)_{t}^{p, \mathbb{F}}\right|_{u=\xi}\right)=\mathbb{E}\left(o, \mathbb{F}\left(\left.\left(\mathbb{1}_{\left[\widetilde{R}^{u}, \infty[\right.}\right)^{p, \mathbb{F}}\right|_{u=\xi}\right)_{t}\right) \\
& =\mathbb{E}\left(\int_{\mathbb{R}}\left(\mathbb{1}_{\left[\widetilde{R}^{u}, \infty[\right.}\right]_{t}^{p, \mathbb{F}} q_{t}^{u} \eta(d u)\right)=\int_{\mathbb{R}} \mathbb{E}\left(\left(\mathbb{1}_{\left[\widetilde{R}^{u}, \infty[\right.}\right)_{t}^{p, \mathbb{F}} q_{t}^{u}\right) \eta(d u),
\end{aligned}
$$

where the second equality comes from (6). Next, by Yoeurp's lemma we conclude that, for each $t$

$$
0=\int_{\mathbb{R}} \mathbb{E}\left(\int_{0}^{t} q_{s-}^{u} d\left(\left(\mathbb{1}_{\left[\widetilde{R}^{u}, \infty[\right.}\right)^{p, \mathbb{F}}\right)_{s}\right) \eta(d u)=\int_{\mathbb{R}} \mathbb{E}\left(q_{\widetilde{R}^{u}-}^{u} \mathbb{1}_{\left\{\tilde{R}^{u} \leq t\right\}}\right) \eta(d u)
$$

which in turn is equivalent to $\widetilde{R}^{u}>t, \mathbb{P} \otimes \eta$-a.s. for each $t$ since $q_{\widetilde{R}_{-}}^{u}>0$. Thus, $\frac{1}{q^{\xi}}$ is an $\mathbb{F}^{\sigma(\xi)}$-local martingale if and only if $\widetilde{R}^{u}$ is infinite $\mathbb{P} \otimes \eta$-a.s.

(c) The "if" part is shown in Theorem 1. We show "only if" part here. Assume that the process $\frac{1}{q^{\xi}}$ is a true $\mathbb{F}^{\sigma(\xi)}$-martingale. Then, for each $t \geq 0$, we have $\mathbb{E}\left(\frac{1}{q_{t}^{\xi}}\right)=1$. On the other hand, using Lemma 1 (ii), we have that

$\mathbb{E}\left(\frac{1}{q_{t}^{\xi}}\right)=\mathbb{E}\left(\int_{\mathbb{R}} \frac{1}{q_{t}^{u}} \mathbb{1}_{\left\{q_{t}^{u}>0\right\}} q_{t}^{u} \eta(d u)\right)=\int_{\mathbb{R}} \mathbb{P}\left(q_{t}^{u}>0\right) \eta(d u)=\int_{\mathbb{R}} \mathbb{P}\left(R^{u}>t\right) \eta(d u)$,

which shows that $R^{u}=\infty, \mathbb{P} \otimes \eta$-a.s..

In [6], Amendinger establishes that under equivalence Jacod's hypothesis, for any $\mathbb{F}$-martingale $X$, the process $X / q^{\xi}$ is a $\mathbb{G}$ martingale. In the following proposition, we investigate the $\mathbb{F}^{\sigma(\xi)}$-semimartingale decomposition of a parametrized $\mathbb{F}$-local martingale $X$ when $\xi$ is plugged in and when multiplied by $\frac{1}{q^{\xi}}$ from previous lemma.

Proposition 9. Let $\left(X^{u}, u \in \mathbb{R}\right)$ be a parametrized $\mathbb{F}$-local martingale. Then $\frac{X^{\xi}}{q^{\xi}}$ is an $\mathbb{F}^{\sigma(\xi)}$-semimartingale with $\mathbb{F}^{\sigma(\xi)}$-local martingale part equal to

$$
X_{0}^{\xi}-\frac{X_{-}^{\xi}}{\left(q^{\xi}\right)_{-}^{2}} \cdot \bar{q}^{\xi}+\frac{1}{q_{-}^{\xi}} \cdot \bar{X}^{\xi}
$$

and $\mathbb{F}^{\sigma(\xi)}$-predictable finite variation part equal to

$$
-\left.\frac{1}{q_{-}^{\xi}} \cdot\left(X_{\widetilde{R}^{u}}^{u} \mathbb{1}_{\left[\widetilde{R}^{u}, \infty[\right.}\right)^{p, \mathbb{F}}\right|_{u=\xi} \cdot
$$

Proof. We compute, applying integration by parts formula: 


$$
\begin{aligned}
\frac{X^{\xi}}{q^{\xi}}= & X_{0}^{\xi}+X_{-}^{\xi} \cdot \frac{1}{q^{\xi}}+\frac{1}{q_{-}^{\xi}} \cdot X^{\xi}+\left[X^{\xi}, \frac{1}{q^{\xi}}\right] \\
= & X_{0}^{\xi}-\frac{X_{-}^{\xi}}{\left(q^{\xi}\right)_{-}^{2}} \cdot \bar{q}^{\xi}-\left.\frac{X_{-}^{\xi}}{q_{-}^{\xi}} \cdot\left(\mathbb{1}_{\left[\widetilde{R}^{u}, \infty[\right.}\right)^{p, \mathbb{F}}\right|_{u=\xi} \\
& +\frac{1}{q_{-}^{\xi}} \cdot \bar{X}^{\xi}+\frac{1}{q_{-}^{\xi} q^{\xi}} \cdot\left[X^{\xi}, q^{\xi}\right]-\left.\frac{1}{q_{-}^{\xi}} \cdot\left(\Delta X_{\widetilde{R}^{u}}^{u} \mathbb{1}_{\widetilde{R}^{u}, \infty}\right)^{p, \mathbb{F}}\right|_{u=\xi} \\
& -\frac{1}{\left(q^{\xi}\right)_{-}^{2}} \cdot\left[X^{\xi}, q^{\xi}\right]+\frac{1}{\left(q^{\xi}\right)_{-}^{2} q^{\xi}} \cdot\left[X^{\xi},\left[q^{\xi}\right]\right],
\end{aligned}
$$

where the second equality comes from (16). It follows that

$$
\begin{aligned}
\frac{X^{\xi}}{q^{\xi}}= & X_{0}^{\xi}-\frac{X_{-}^{\xi}}{\left(q^{\xi}\right)_{-}^{2}} \cdot \bar{q}^{\xi}+\frac{1}{q_{-}^{\xi}} \cdot \bar{X}^{\xi} \\
& -\left.\frac{1}{q_{-}^{\xi}} \cdot\left(X_{\widetilde{R}^{u}}^{u} \mathbb{1}_{\left[\widetilde{R}^{u}, \infty[\right.}\right)^{p, \mathbb{F}}\right|_{u=\xi}-\frac{\Delta q^{\xi}}{\left.p_{(} \xi\right)^{2} q^{\xi}} \cdot\left[X^{\xi}, q^{\xi}\right]+\frac{\Delta X^{\xi}}{\left(q^{\xi}\right)_{-}^{2} q^{\xi}} \cdot\left[q^{\xi}\right] \\
= & X_{0}^{\xi}-\frac{X_{-}^{\xi}}{\left(q^{\xi}\right)_{-}^{2}} \cdot \bar{q}^{\xi}+\frac{1}{q_{-}^{\xi}} \cdot \bar{X}^{\xi}-\left.\frac{1}{q_{-}^{\xi}} \cdot\left(X_{\widetilde{R}^{u}}^{u} \mathbb{1}_{\left[\widetilde{R}^{u}, \infty[\right.}\right)^{p, \mathbb{F}}\right|_{u=\xi} \cdot
\end{aligned}
$$

As a corollary, from Proposition 9, we recover [16, Proposition 5.2] on universal supermartingale density.

Corollary 1. If $X$ is a positive $\mathbb{F}$-supermartingale, then, $\frac{X}{q^{\xi}}$ is an $\mathbb{F}^{\sigma(\xi)}$-supermartingale.

Proof. Let $X$ be decomposed as $X=M^{X}-V^{X}$ where $M^{X}$ is a positive $\mathbb{F}$-local martingale and $V^{X}$ is an increasing $\mathbb{F}$-predictable process. Then, $\frac{M^{X}}{q^{\xi}}$ is an $\mathbb{F}^{\sigma(\xi)}$ supermartingale since from the positiveness of $M^{X}$, by Proposition 9, we get that $\left.\frac{1}{q_{-}^{\xi}} \cdot\left(M_{\widetilde{R}^{u}}^{X} \mathbb{1}_{\left[\widetilde{R}^{u}, \infty[\right.}\right)^{p, \mathbb{F}}\right|_{u=\xi}$ is an $\mathbb{F}^{\sigma(\xi)}$-predictable increasing process. Moreover, as $\frac{1}{q^{\xi}}$ is an $\mathbb{F}^{\sigma(\xi)}$-supermartingale and $V^{X}$ is predictable and increasing, the process $-\frac{V^{X}}{q^{\xi}}$ is as well an $\mathbb{F}^{\sigma(\xi)}$-supermartingale which ends the proof.

\subsection{NUPBR condition for initial enlargement}

In this section, we focus on the NUPBR condition in an initial enlargement framework. Using simple arguments based on our optional semimartingale decomposition, we prove the stability of the NUPBR condition with respect to an initial enlargement of filtration under absolute continuity Jacod's hypothesis. In Proposition 10 , we give $\mathbb{F}^{\sigma(\xi)}$-local martingale deflators for quasi left-continuous parametrized 
$\mathbb{F}$-local martingales and $\mathbb{F}^{\sigma(\xi)}$-supermartingale deflators for parametrized $\mathbb{F}$-local martingales. In Theorem 6 , we present a necessary and sufficient condition such that any parametrized $\mathbb{F}$-local martingale satisfies $\operatorname{NUPBR}\left(\mathbb{F}^{\sigma(\xi)}\right)$. We close this section by giving two particular examples of initial enlargements under Jacod's hypothesis. We address the reader to [1] for a study similar to the one contained in this section using fully different methodology.

We denote by $\widetilde{R}^{u, a}$ the accessible part of the $\mathbb{F}$-stopping time $\widetilde{R}^{u}$ and we define the process $V^{\mathrm{i}}$ as

$$
V_{t}^{\mathrm{i}}:=\sum_{0 \leq s \leq t} p,\left.\mathbb{F}\left(\mathbb{1}_{\left[\widetilde{R}^{u}\right]}\right)_{s}\right|_{u=\xi}=\left.\left(\mathbb{1}_{\left[\widetilde{R}^{u, a}, \infty[\right.}\right)^{p, \mathbb{F}}\right|_{u=\xi} .
$$

Proposition 10. Let $L^{\mathrm{i}}$ be defined in (15), and let $\left(X^{u}, u \in \mathbb{R}\right)$ be a parametrized $\mathbb{F}$-local martingale such that $\Delta X_{\widetilde{R}^{u}}^{u}=0$ on $\left\{\widetilde{R}^{u}<\infty\right\} \mathbb{P} \otimes \eta$-a.s. .

(a) If $\left(X^{u}, u \in \mathbb{R}\right)$ is quasi-left continuous, then the process $Y^{\mathrm{i}}:=\mathscr{E}\left(-L^{\mathrm{i}}\right)$ is an $\mathbb{F}^{\sigma(\xi)}$-local martingale deflator for $X^{\xi}$.

(b) In general, the process $\widetilde{Y}^{\mathrm{i}}:=\mathscr{E}\left(-L^{\mathrm{i}}-V^{\mathrm{i}}\right)$ is an $\mathbb{F}^{\sigma(\xi)}$-supermartingale deflator for $X^{\xi}$.

Proof. (a) Using the optional decomposition (14) given in Theorem 5, firstly for $X^{\xi}$ and then for $q^{\xi}$, we obtain

$$
\begin{aligned}
Y^{\mathrm{i}} X^{\xi} & =X_{-}^{\xi} \cdot Y^{\mathrm{i}}+Y_{-}^{\mathrm{i}} \cdot X^{\xi}+\left[Y^{\mathrm{i}}, X^{\xi}\right] \\
& =X_{-}^{\xi} \cdot Y^{\mathrm{i}}+Y_{-}^{\mathrm{i}} \cdot \bar{X}^{\xi}+Y_{-}^{\mathrm{i}} \frac{1}{q^{\xi}} \cdot\left[X^{\xi}, q^{\xi}\right]-\left.Y_{-}^{\mathrm{i}} \cdot\left(\Delta X_{\widetilde{R}^{u}}^{u}\right)^{p, \mathbb{F}}\right|_{u=\xi}-Y_{-}^{\mathrm{i}} \cdot\left[L^{\mathrm{i}}, X^{\xi}\right] \\
& =X_{-}^{\xi} \cdot Y^{\mathrm{i}}+Y_{-}^{\mathrm{i}} \cdot \bar{X}^{\xi}+Y_{-}^{\mathrm{i}} \frac{1}{q^{\xi}} \cdot\left[X^{\xi}, \bar{q}^{\xi}\right]+Y_{-}^{\mathrm{i}} \frac{1}{\left(q^{\xi}\right)^{2}} \cdot\left[\left[q^{\xi}\right], X^{\xi}\right] \\
& -Y_{-}^{\mathrm{i}} \frac{1}{q^{\xi}} \cdot\left[\left.\left(\Delta q_{\widetilde{R}_{-}^{u}}^{u} \mathbb{1}_{\left[\widetilde{R}^{u}, \infty[\right.}\right)^{p, \mathbb{F}}\right|_{u=\xi}, X^{\xi}\right]-\left.Y_{-}^{\mathrm{i}} \cdot\left(\Delta X_{\widetilde{R}^{u}}^{u} \mathbb{1}_{\left[\widetilde{R}^{u}, \infty[\right.}\right)^{p, \mathbb{F}}\right|_{u=\xi}-\frac{Y_{-}^{\mathrm{i}}}{q_{-}^{\xi}} \cdot\left[X^{\xi}, \bar{q}^{\xi}\right] .
\end{aligned}
$$

We continue, computing the various brackets:

$$
\begin{aligned}
Y^{\mathrm{i}} X^{\xi} & =X_{-}^{\xi} \cdot Y^{\mathrm{i}}+Y_{-}^{\mathrm{i}} \cdot \bar{X}^{\xi}-\sum \frac{Y_{-}^{\mathrm{i}} \Delta q^{\xi}}{q^{\xi} q_{-}^{\xi}} \Delta X^{\xi} \Delta \bar{q}^{\xi}+\sum \frac{Y_{-}^{\mathrm{i}}}{\left(q^{\xi}\right)^{2}}\left(\Delta q^{\xi}\right)^{2} \Delta X^{\xi} \\
& +\sum \frac{Y_{-}^{\mathrm{i}} q_{-}^{\xi}}{q^{\xi}} p,\left.\mathbb{F}\left(\mathbb{1}_{\left[\widetilde{R}^{u}\right]}\right)\right|_{u=\xi} \Delta X^{\xi}-\left.Y_{-}^{\mathrm{i}} \cdot\left(\Delta X_{\widetilde{R}^{u}}^{u} \mathbb{1}_{\left[\widetilde{R}^{u}, \infty[\right.}\right)^{p, \mathbb{F}}\right|_{u=\xi} \\
& =X_{-}^{\xi} \cdot Y^{\mathrm{i}}+Y_{-}^{\mathrm{i}} \cdot \bar{X}^{\xi}+\sum Y_{-}^{\mathrm{i}} p,\left.\mathbb{F}\left(\mathbb{1}_{\left[\widetilde{R}^{u}\right]}\right)\right|_{u=\xi} \Delta X^{\xi}-\left.Y_{-}^{\mathrm{i}} \cdot\left(\Delta X_{\widetilde{R}^{u}}^{u} \mathbb{1}_{\left[\widetilde{R}^{u}, \infty\right]}\right)^{p, \mathbb{F}}\right|_{u=\xi} \\
& =X_{-}^{\xi} \cdot Y^{\mathrm{i}}+Y_{-}^{\mathrm{i}} \cdot \bar{X}^{\xi}+\left.\sum Y_{-}^{\mathrm{i}} p \mathbb{F}^{(\mathbb{F}}\left(\mathbb{1}_{\left[\widetilde{R}^{u}\right]}\right)\right|_{u=\xi} \Delta X^{\xi}+\left.\frac{Y_{-}^{\mathrm{i}}}{q_{-}^{\xi}} \cdot\left(\mathbb{1}_{\left[\widetilde{R}^{u}\right]} \cdot\left[X^{u}, q^{u}\right]\right)^{p, \mathbb{F}}\right|_{u=\xi} \cdot
\end{aligned}
$$

where the last equality follows from $\Delta q_{\widetilde{R}^{u}}^{u}=-q_{\widetilde{R}_{-}}^{u}$ on $\left\{\widetilde{R}^{u}<\infty\right\}$. 
Since $\left(X^{u}, u \in \mathbb{R}\right)$ is an $\mathbb{F}$-quasi-left continuous local martingale and $\Delta X_{R^{u}}^{u}=0$ on $\left\{R^{u}<\infty\right\}$, the two last terms are null, and $Y^{\mathrm{i}} X^{\xi}$ is an $\mathbb{F}^{\sigma(\xi)}$-local martingale. Therefore, $Y^{\mathrm{i}}$ is an $\mathbb{F}^{\sigma(\xi)}$-local martingale deflator for $X^{\xi}$.

(b) Let $H$ be an $\mathbb{F}^{\sigma(\xi)}$-predictable process such that $H \cdot X^{\xi} \geq-1$. Then, by integration by parts, we get

$$
\left(1+H \cdot X^{\xi}\right) \widetilde{Y}^{\mathrm{i}}=\left(1+H \cdot X^{\xi}\right)_{-} \cdot \widetilde{Y}^{\mathrm{i}}+H \widetilde{Y}_{-}^{\mathrm{i}} \cdot X^{\xi}-H \widetilde{Y}_{-}^{\mathrm{i}} \cdot\left[X^{\xi}, L^{\mathrm{i}}\right]-H \widetilde{Y}_{-}^{\mathrm{i}} \cdot\left[X^{\xi}, V^{\mathrm{i}}\right] .
$$

Note that

$$
H \widetilde{Y}_{-}^{\mathrm{i}} \cdot\left[X^{\xi}, V^{\mathrm{i}}\right]=\left.\sum H \widetilde{Y}_{-}^{\mathrm{i} p, \mathbb{F}}\left(\mathbb{1}_{\left[\widetilde{R}^{u}\right]}\right)\right|_{u=\xi} \Delta X^{\xi} .
$$

Then, using the same arguments as in the proof of Theorem 10, we get

$$
\left(1+H \cdot X^{\xi}\right) \widetilde{Y}^{\mathrm{i}}=\left(1+H \cdot X^{\xi}\right)_{-} \cdot \widetilde{Y}^{\mathrm{i}}+H \widetilde{Y}_{-}^{\mathrm{i}} \cdot \bar{X}^{\xi}-\left.H \widetilde{Y}_{-}^{\mathrm{i}}\left(\Delta X_{\widetilde{R}^{u}}^{u} \mathbb{1}_{\left[\widetilde{R}^{u}, \infty \mathrm{l}\right.}\right)^{p, \mathbb{F}}\right|_{u=\xi}
$$

and the assertion is proved.

Proposition 11. Let $\left(X^{u}, u \in \mathbb{R}\right)$ be a parametrized process admitting an $\mathbb{F}$-local martingale deflator such that $\Delta X_{\widetilde{R}^{u}}^{u}=0$ on $\left\{\widetilde{R}^{u}<\infty\right\}$, $\eta$-a.e. Then $X^{\xi}$ admits an $\mathbb{F}^{\sigma(\xi)}$-local martingale deflator.

Proof. Let $\left(X^{u}, u \in \mathbb{R}\right)$ be a parametrized $\mathbb{F}$-semimartingale admitting an $\mathbb{F}$-local martingale deflator, i.e., there exist a real-valued parametrized predictable process $\left(\phi^{u}, u \in \mathbb{R}\right)$ and a positive $\mathbb{F}$-local martingale $L$ such that

$$
0<\phi^{u} \leq 1 \quad \text { and } \quad\left(L\left(\phi^{u} \cdot X^{u}\right), u \in \mathbb{R}\right) \quad \text { is a parametrized } \mathbb{F} \text {-local martingale. }
$$

Then, there exists a sequence of $\mathbb{F}$-stopping times that increases to infinity $\left(T_{n}\right)_{n}$ such that $L^{T_{n}}$ is a martingale. Put $\mathbb{Q}_{n}:=L_{T_{n}} \cdot P \sim P$. Then, by applying Proposition 10 to $\left(\left(\phi^{u} \cdot X^{u}\right)^{T_{n}}, u \in \mathbb{R}\right)$ under $\mathbb{Q}_{n}$, we conclude that $\left(\phi^{\xi} \cdot X^{\xi}\right)^{T_{n}}$ satisfies $\operatorname{NUPBR}\left(\mathbb{F}^{\sigma(\xi)}\right)$ under $\mathbb{Q}_{n}$. Thanks to Proposition 5 , NUPBR $\left(\mathbb{F}^{\sigma(\xi)}\right)$ property under $\mathbb{P}$ of $X^{\xi}$ follows immediately.

Theorem 6. The following conditions are equivalent.

(a) The thin set $\left\{q^{u}=0<q_{-}^{u}\right\}$ is evanescent $\eta$-a.e.

(b) The $\mathbb{F}$-stopping time $\widetilde{R}^{u}$ is infinite $\mathbb{P} \otimes \eta$-a.s.

(c) For any parametrized $\mathbb{F}$-local martingale $\left(X^{u}, u \in \mathbb{R}\right)$, the process $X^{\xi}$ admits an $\mathbb{F}^{\sigma(\xi)}$-local martingale deflator $\frac{1}{q^{\xi}}$ (and satisfies $N U P B R\left(\mathbb{F}^{\sigma(\xi)}\right)$ ).

(d) For any parametrized (bounded) process $\left(X^{u}, u \in \mathbb{R}\right)$ admitting an $\mathbb{F}$-local martingale deflator, the process $X^{\xi}$ admits an $\mathbb{F}^{\sigma(\xi)}$-local martingale deflator (and satisfies $N U P B R\left(\mathbb{F}^{\sigma(\xi)}\right)$ ).

Proof. The equivalence between (a) and (b) is obvious from the definition of $\widetilde{R}^{u}$.

The implication (b) $\Rightarrow$ (c) follows from Proposition 10 . To prove (c) $\Rightarrow(b)$, we consider a parametrized $\mathbb{F}$-martingale $\left(M^{u}, u \in \mathbb{R}\right)$ with

$$
M^{u}:=\mathbb{1}_{\left[\widetilde{R}^{u}, \infty[\right.}-\left(\mathbb{1}_{\left[\widetilde{R}^{u}, \infty\right]}\right)^{p, \mathbb{F}} .
$$


Then, due to the equality $R^{\xi}=\infty$ established in (7), it is clear that

$$
M^{\xi}=-\left.\left(\mathbb{1}_{\left[\widetilde{R}^{u}, \infty[\right.}\right)^{p, \mathbb{F}}\right|_{u=\xi}
$$

is decreasing. Thus, $M^{\xi}$ satisfies $\operatorname{NUPBR}\left(\mathbb{F}^{\sigma(\xi)}\right)$ if and only if it is a null process. Then, we conclude that $\widetilde{R}$ is infinite using the same argument as in the proof of Lemma 5 (b). The implication (c) $\Rightarrow$ (d) follows from Proposition 11.

In the two following examples we look at two extreme situations.

Example 1. Let $\mathbb{F}$ be a filtration such that each $\mathbb{F}$-martingale is continuous. Then, the NUPBR condition is preserved in an initially enlarged filtration for any parametrized $\mathbb{F}$-local martingale from the reference filtration.

Example 2. Let $B$ be a $\mathscr{G}$-measurable set such that $\mathbb{P}(B)=\frac{1}{2}$ and consider the filtration $\mathbb{F}=\left(\mathscr{F}_{t}\right)_{t \geq 0}$ defined as

$$
\mathscr{F}_{t}=\{\emptyset, \Omega\} \text { for } t \in\left[0,1\left[\text { and } \mathscr{F}_{t}:=\left\{\emptyset, B, B^{c}, \Omega\right\} \text { for } t \in[1, \infty[.\right.\right.
$$

Define a random variable $\xi$ as $\xi:=\mathbb{1}_{B}+2 \cdot \mathbb{1}_{B^{c}}$. The random variable $\xi$ satisfies Jacod's hypothesis with density $\left(q^{u}, u \in\{1,2\}\right)$ equal to

$$
\begin{aligned}
& q^{1}=\mathbb{1}_{[0,1[}+2 \cdot \mathbb{1}_{\{\xi=1\}} \mathbb{1}_{[1, \infty[}, \\
& q^{2}=\mathbb{1}_{[0,1[}+2 \cdot \mathbb{1}_{\{\xi=2\}} \mathbb{1}_{[1, \infty[} .
\end{aligned}
$$

Let the filtration $\mathbb{F}^{\sigma(\xi)}=\left(\mathscr{F}_{t}^{\sigma(\xi)}\right)_{t \geq 0}$ be an initial enlargement of the filtration $\mathbb{F}$ with $\xi$, i.e.,

$$
\mathscr{F}_{t}{ }^{\sigma(\xi)}:=\left\{\emptyset, B, B^{c}, \Omega\right\} \quad \text { for } \quad t \in[0, \infty[.
$$

Let $X$ be an $\mathbb{F}$-martingale defined as

$$
X:=\left(\mathbb{1}_{\{\xi=1\}}-\frac{1}{2}\right) \mathbb{1}_{[1, \infty[} .
$$

Then, $X$ is an $\mathbb{F}^{\sigma(\xi)}$-predictable process. Thus, by [5, Lemma 2.6] it does not satisfy $\operatorname{NUPBR}\left(\mathbb{F}^{\sigma(\xi)}\right)$. Note that here, the set $\left\{q^{u}=0<q_{-}^{u}\right\}$ is not evanescent, and that $\widetilde{R}^{u}$ is not equal to infinity.

\section{Connection to Absolutely Continuous Change of Measure}

In this section, we study the relationship between our optional semimartingale decompositions in progressive and initial enlargement of filtration cases and our optional semimartingale decomposition in an absolutely continuous change of measure set-up. First let us recall [28, Theorem 42, Chapter III]. 
Theorem 7. Let $X$ be a $\mathbb{P}$-local martingale with $X_{0}=0$. Let $\mathbb{Q}$ be a probability measure absolutely continuous with respect to $\mathbb{P}$, and define $\zeta_{t}:=\mathbb{E}_{\mathbb{P}}\left(\frac{d \mathbb{Q}}{d \mathbb{P}} \mid \mathscr{F}_{t}\right)$. Let $r:=\inf \left\{t>0: \zeta_{t}=0\right\}$ and $\widetilde{r}:=r_{\left\{\zeta_{r-}>0\right\}}$. Then

$$
\bar{X}:=X-\frac{1}{\zeta} \cdot[X, \zeta]+\left(\Delta X_{\widetilde{r}} \mathbb{1}_{[\widetilde{r}, \infty[}\right)^{p, \mathbb{P}}
$$

is a $\mathbb{Q}$-local martingale.

It is clear that Theorem 7 implies the same type of decompositions as the two decompositions stated in Sections 2.1 and 3.1.

$$
\begin{array}{ll}
\text { Up to random time } \tau: & X^{\tau}=\bar{X}+\frac{1}{N^{\tau}} \cdot\left[X^{\tau}, N^{\tau}\right]-\left(\Delta X_{\widetilde{R}} \mathbb{1}_{[\widetilde{R}, \infty[}\right)^{p, \mathbb{F}} \\
\text { Under Jacod's hypothesis: } & X^{\xi}=\bar{X}+\frac{1}{q^{\xi}} \cdot\left[X^{\xi}, q^{\xi}\right]-\left.\left(\Delta X_{\widetilde{R}^{u}}^{u} \mathbb{1}_{\left[\widetilde{R}^{u}, \infty[\right.}\right)^{p, \mathbb{F}}\right|_{u=\xi} \\
\text { Under measure } \mathbb{Q}: & X=\bar{X}+\frac{1}{\zeta} \cdot[X, \zeta]-\left(\Delta X_{\widetilde{r}} \mathbb{1}_{[\widetilde{r}, \infty[}\right)^{p, \mathbb{P}}
\end{array}
$$

In each of the three cases, there is a different mechanism to ensure the strict positivity of $N^{\tau}, q^{\xi}$ and $\zeta$. In the case of progressive enlargement up to a random time, we stop at $\tau$. In the case of initial enlargement with random variable satisfying Jacod's hypothesis, we plug $\xi$. In the case of absolutely continuous change of measure, the process $\zeta$ is strictly positive $\mathbb{Q}$-a.s.

The optional decomposition in the change of measure case can be used in the same way to obtain similar result on stability of the NUPBR condition with respect to absolutely continuous change of measure.

We remark here that the set introduced in Definition 3 may become bigger under absolutely continuous change of measure as under the new measure the condition $H \cdot X \geq-1$ is more likely satisfied.

Let $\bar{\zeta}$ given by (18) in terms of the Radon Nikodym density $\zeta$, and define a $\mathbb{Q}$-local martingale $L^{a}$ by

$$
L^{a}:=\frac{1}{\zeta} \cdot \bar{\zeta}
$$

Let us denote by $\widetilde{r}^{a}$ the accessible part of the stopping time $\widetilde{r}$, and set

$$
V^{a}:=\left(\mathbb{1}_{\left[\widetilde{r}^{a}, \infty[\right.}\right)^{p, \mathbb{P}}
$$

Using the processes $L^{a}$ and $V^{a}$ we study, in the next lemma, the behaviour of particular $\mathbb{Q}$-deflators.

Proposition 12. (a) Let $Y^{a}:=\mathscr{E}\left(-L^{a}\right)$. If $X$ is a quasi-left continuous local martingale and $\Delta X_{\widetilde{r}}=0$ on $\{\widetilde{r}<\infty\} \mathbb{P}$-a.s., then $Y^{a}$ is a $\mathbb{Q}$-local martingale deflator for $X$. (b) Let $\widetilde{Y}^{a}:=\mathscr{E}\left(-L^{a}-V^{a}\right)$. Let $X$ be a $\mathbb{P}$-local martingale such that $\Delta X_{\widetilde{r}}=0$ on $\{\widetilde{r}<\infty\} \mathbb{P}$-a.s., then $\widetilde{Y}^{a}$ is a $\mathbb{Q}$-supermartingale deflator for $X$.

Proof. (a) Using integration by parts and the optional decomposition (18) given in Theorem 7 for $X$ and then for $\zeta$, we obtain 


$$
\begin{aligned}
Y^{a} X & =X_{-} \cdot Y^{a}+Y_{-}^{a} \cdot X+\left[Y^{a}, X\right] \\
& =X_{-} \cdot Y^{a}+Y_{-}^{a} \cdot \bar{X}+Y_{-}^{a} \frac{1}{\zeta} \cdot[\zeta, X]-Y_{-}^{a} \cdot\left(\Delta X_{\widetilde{r}} \mathbb{1}_{[\widetilde{r}, \infty[}\right)^{p, \mathbb{P}}-Y_{-}^{a} \cdot\left[L^{a}, X\right] \\
& =X_{-} \cdot Y^{a}+Y_{-}^{a} \cdot \bar{X}+Y_{-}^{a} \frac{1}{\zeta} \cdot[\bar{\zeta}, X]+Y_{-}^{a} \frac{1}{\zeta^{2}}[[\zeta], X] \\
& -Y_{-}^{a} \frac{1}{\zeta} \cdot\left[\left(\Delta \zeta_{\widetilde{r}} \mathbb{1}_{[\widetilde{r}, \infty[}\right)^{p, \mathbb{P}}, X\right]-Y_{-}^{a} \cdot\left(\Delta X_{\widetilde{r}} \mathbb{1}_{[\widetilde{r}, \infty[}\right)^{p, \mathbb{P}}-\frac{Y_{-}^{a}}{\zeta}[\bar{\zeta}, X] .
\end{aligned}
$$

We continue, adding the two terms which contain $[\bar{\zeta}, X]$ and computing the brackets

$$
\begin{aligned}
Y^{a} X & =X_{-} \cdot Y^{a}+Y_{-}^{a} \cdot \bar{X}-\sum \frac{Y_{-}^{a} \Delta \zeta}{\zeta \zeta} \Delta X \Delta \bar{\zeta}+\sum \frac{Y_{-}^{a}}{\zeta^{2}}(\Delta \zeta)^{2} \Delta X \\
& +\sum \frac{Y_{-}^{a} \zeta_{-}}{\zeta}{ }^{p, \mathbb{P}}\left(\mathbb{1}_{[\tilde{r}]}\right) \Delta X-Y_{-}^{a} \cdot\left(\Delta X_{\widetilde{r}} \mathbb{1}_{[\widetilde{r}, \infty[}\right)^{p, \mathbb{P}} \\
& =X_{-} \cdot Y^{a}+Y_{-}^{a} \cdot \bar{X}+\sum Y_{-}^{a} p, \mathbb{P}\left(\mathbb{1}_{[\tilde{r}]}\right) \Delta X-Y_{-}^{a} \cdot\left(\Delta X_{\widetilde{r}} \mathbb{1}_{[\widetilde{r}, \infty[}\right)^{p, \mathbb{P}}
\end{aligned}
$$

Since for any $\mathbb{P}$-quasi-left continuous martingale $X$, the process $p, \mathbb{P}\left(\mathbb{1}_{[\tilde{r}]}\right) \Delta X$ is null and $\Delta X_{\widetilde{r}}=0, Y^{a}$ is a $\mathbb{Q}$-local martingale deflator for $X$.

(b) Let $H$ be a predictable process such that $H . X \geq-1$. Then, by integration by parts, we get

$$
(1+H \cdot X) \tilde{Y}^{a}=(1+H \cdot X)_{-} \cdot \widetilde{Y}^{a}+H \widetilde{Y}_{-}^{a} \cdot X-H \widetilde{Y}_{-}^{a} \cdot\left[X, L^{a}\right]-H \widetilde{Y}_{-}^{a} \cdot\left[X, V^{a}\right] .
$$

Note that

$$
H \widetilde{Y}_{-}^{a} \cdot\left[X, V^{a}\right]=\sum H \widetilde{Y}_{-}^{a}{ }^{p, \mathbb{P}}\left(\mathbb{1}_{[\tilde{r}]}\right) \Delta X .
$$

Then, using the same arguments as in the proof of Theorem 12 to derive (19), we get

$$
(1+H \cdot X) \widetilde{Y}^{a}=(1+H \cdot X)_{-} \cdot \widetilde{Y}^{a}+H \widetilde{Y}_{-}^{a} \cdot \bar{X}-H \widetilde{Y}_{-}^{a} \cdot\left(\Delta X_{\widetilde{r}} \mathbb{1}_{[\widetilde{S}, \infty[}\right)^{p, \mathbb{P}}
$$

and the assertion is proved.

Proposition 13. Let $X$ be a process admitting a $\mathbb{P}$-local martingale deflator such that $\Delta X_{\widetilde{\zeta}}=0$ on $\{\widetilde{\zeta}<\infty\}$. Then $X$ admits a $\mathbb{Q}$-local martingale deflator.

Proof. Let $X$ be an $\mathbb{P}$-semimartingale satisfying NUPBR $(\mathbb{P})$. Thanks to Proposition 5 and Theorem 2, we deduce the existence of a real-valued predictable process $\phi$ and a positive $\mathbb{P}$-local martingale $K$ such that

$$
0<\phi \leq 1 \quad \text { and } \quad K(\phi \cdot X) \quad \text { is a } \mathbb{P} \text {-local martingale. }
$$

Then there exists a sequence of stopping times $\left(v_{n}\right)_{n}$ that increases to infinity such that the stopped process $K^{v_{n}}$ is a $\mathbb{P}$-martingale. Put $\mathbb{P}_{n}:=K_{v_{n}} . \mathbb{P} \sim \mathbb{P}$ and 


$$
\mathbb{Q}_{n}:=\frac{K_{v_{n}}}{\mathbb{E}_{\mathbb{P}}\left(\zeta_{v_{n}} K_{v_{n}}\right)} \cdot \mathbb{Q}=\frac{\zeta_{v_{n}}}{\mathbb{E}_{\mathbb{P}}\left(\zeta_{v_{n}} K_{v_{n}}\right)} \cdot \mathbb{P}_{n} \ll \mathbb{P}_{n}
$$

Define $\zeta_{t}^{n}:=\mathbb{E}_{\mathbb{P}_{n}}\left(\frac{\zeta_{v_{n}}}{\mathbb{E}_{\mathbb{P}_{n}}\left(\zeta_{v_{n}}\right)} \mid \mathscr{F}_{t}\right)$ and note that the condition that $\left\{\zeta=0<\zeta_{-}\right\}$is $\mathbb{P}_{-}$ evanescent implies that $\left\{\zeta^{n}=0<\zeta_{-}^{n}\right\}$ is $\mathbb{P}_{n}$-evanescent. Then, by applying Proposition 12 to $(\phi \cdot X)^{v_{n}}$ under $\mathbb{P}_{n}$, we conclude that $(\phi \cdot X)^{v_{n}}$ satisfies $\operatorname{NUPBR}\left(\mathbb{Q}_{n}\right)$. Thanks to Proposition 5 , since $\mathbb{Q}_{n} \sim \mathbb{Q}, \operatorname{NUPBR}(\mathbb{Q})$ property of $X$ immediately follows.

We recover [10, Theorem 5.3] and [10, Proposition 5.7] with alternative proof in the next result.

Theorem 8. The following conditions are equivalent.

(a) The thin set $\left\{\zeta=0<\zeta_{-}\right\}$is $\mathbb{P}$-evanescent.

(b) The stopping time $\widetilde{r}$ is infinite $\mathbb{P}$-a.s.

(c) Any $\mathbb{P}$-local martingale $X$ admits $Y^{a}$ as a $\mathbb{Q}$-local martingale deflator, so $X$ satisfies $N U P B R(\mathbb{Q})$.

(d) Any (bounded) process $X$ satisfying $\operatorname{NUPBR}(\mathbb{P})$ satisfies $\operatorname{NUPBR}(\mathbb{Q})$.

Proof. The equivalence between (a) and (b) is obvious from the definition of $\widetilde{r}$.

The implication $(b) \Rightarrow(c)$ follows from Proposition 12. To prove $(c) \Rightarrow(b)$ (and $(\mathrm{d}) \Rightarrow(\mathrm{b}))$, we consider the $\mathbb{P}$-martingale

$$
X=\mathbb{1}_{[\widetilde{r}, \infty[}-\left(\mathbb{1}_{\left[\widetilde{r}, \infty[)^{p, \mathbb{P}}\right.} .\right.
$$

Then, due to $\widetilde{r}=\infty \mathbb{Q}$-a.s. we have that, under $\mathbb{Q}$,

$$
X=-\left(\mathbb{1}_{[\widetilde{r}, \infty[}\right)^{p, \mathbb{P}}
$$

is a predictable decreasing process. Thus, $X$ satisfies $\operatorname{NUPBR}(\mathbb{Q})$ if and only if it is a null process. Then, we conclude that $\widetilde{S}$ is infinite using the same argument as in the proof of Lemma 6 (b). The implication (c) $\Rightarrow$ (d) follows from Proposition 13.

Acknowledgements The authors are thankful to the Chaire Marchés en Mutation (Fédération Bancaire Française) for financial support and to Marek Rutkowski for valuable comments that helped to improve this paper.

\section{References}

1. Acciaio, B., Fontana, C., And Kardaras, C. Arbitrage of the first kind and filtration enlargements in semimartingale financial models. Preprint, arXiv:1401.7198 (2014).

2. Aksamit, A., Choulli, T., Deng, J., And Jeanblanc, M. Arbitrages in a progressive enlargement setting. Arbitrage, Credit and Informational Risks, Peking University Series in Mathematics 6 (2014), 55-88. 
3. Aksamit, A., Choulli, T., Deng, J., And Jeanblanc, M. Non-arbitrage under a class of honest times. Preprint, arXiv: 1404.0410 (2014).

4. Aksamit, A., Choulli, T., Deng, J., and Jeanblanc, M. Non-Arbitrage up to Random Horizon for Semimartingale Models, long version. Preprint, arXiv: 1310.1142v2 (2014).

5. Aksamit, A., Choulli, T., Deng, J., And Jeanblanc, M. Non-Arbitrage up to Random Horizon for Semimartingale Models, short version. Preprint, arXiv:1310.1142 (2014).

6. AmENDINGER, J. Initial enlargement of filtrations and additional information in financial markets. PhD thesis, Technischen Universiteit Berlin (1999).

7. Choulli, T., Deng, J., AND MA, J. How non-arbitrage, viability and numéraire portfolio are related. Finance and Stochastics, forthcoming (2015).

8. Delbaen, F., And Schachermayer, W. A general version of the fundamental theorem of asset pricing. Mathematische annalen 300, 1 (1994), 463-520.

9. Dellacherie, C., Meyer, P. A., And Maisonneuve, B. Probabilités et potentiel: Chapitres 17 à 24. Processus de Markov (fin), compléments de calcul stochastique. Hermann, 1992.

10. Fontana, C. No-arbitrage conditions and absolutely continuous changes of measure. Arbitrage, Credit and Informational Risks, Peking University Series in Mathematics 6 (2014), 3-18.

11. Fontana, C., Jeanblanc, M., And Song, S. On arbitrages arising from honest times. Finance and Stochastics (2014), 515-543.

12. GRoRUd, A., AND PONTIER, M. Insider trading in a continuous time market model. International Journal of Theoretical and Applied Finance 1, 03 (1998), 331-347.

13. Grorud, A., AND Pontier, M. Asymmetrical information and incomplete markets. International Journal of Theoretical and Applied Finance 4, 02 (2001), 285-302.

14. Hulley, H., AND SChWEIZER, M. M6-on minimal market models and minimal martingale measures. In Contemporary Quantitative Finance. Springer, 2010, pp. 35-51.

15. IMKELleR, P. Random times at which insiders can have free lunches. Stochastics: An International Journal of Probability and Stochastic Processes 74, 1-2 (2002), 465-487.

16. IMKELLER, P., AND PERKOWSKI, N. The existence of dominating local martingale measures. Finance and Stochastics, forthcoming (2015).

17. JACOD, J. Grossissement initial, hypothèse $\left(\mathscr{H}^{\prime}\right)$ et théorème de Girsanov. In Grossissements de filtrations: exemples et applications. Springer, 1985, pp. 15-35.

18. JeAnblanc, M., Yor, M., AND Chesney, M. Mathematical methods for financial markets. Springer, 2009.

19. JEULin, T. Semi-martingales et grossissement d'une filtration. Springer, 1980.

20. Jeulin, T., AND Yor, M. Grossissement d'une filtration et semi-martingales: formules explicites. In Séminaire de Probabilités XII. Springer, 1978, pp. 78-97.

21. Kabanov, Y. On the FTAP of Kreps-Delbaen-Schachermayer. Statistics and control of stochastic processes (Moscow, 1995/1996) (1997), 191-203.

22. Kabanov, Y., Kardaras, C., And Song, S. On local martingale deflators and market portfolios. arXiv:1501.04363 (2014).

23. KARATZAS, I., AND KARDARAS, C. The numéraire portfolio in semimartingale financial models. Finance and Stochastics 11, 4 (2007), 447-493.

24. KARDARAS, C. Market viability via absence of arbitrage of the first kind. Finance and stochastics 16, 4 (2012), 651-667.

25. Kardaras, C. On the stochastic behaviour of optional processes up to random times. The Annals of Applied Probability, forthcoming (2015).

26. LARSEN, K., AND ŽITKOVIĆ, G. On utility maximization under convex portfolio constraints. The Annals of Applied Probability 23, 2 (2013), 665-692.

27. Platen, E. A benchmark approach to finance. Mathematical Finance 16, 1 (2006), 131-151.

28. PROTTER, P. Stochastic Integration and Differential Equations: Version 2.1, vol. 21. Springer Verlag, 2004.

29. RoKhlin, D. B. On the existence of an equivalent supermartingale density for a fork-convex family of stochastic processes. Mathematical Notes 87, 3-4 (2010), 556-563. 
30. Ruf, J. Hedging under arbitrage. Mathematical Finance 23, 2 (2013), 297-317.

31. SCHWEIZER, M., AND TAKAOKA, K. A note on the condition of no unbounded profit with bounded risk. Finance and Stochastics 28, 2 (2013), 393-405.

32. Song, S. Grossissement de filtration et problèmes connexes. PhD thesis, Université Paris VI (1987).

33. SonG, S. Local martingale deflators for asset processes stopped at a default time st or right before st. arXiv preprint arXiv::1405.4474 (2013).

34. STRICKER, C., AND YOR, M. Calcul stochastique dépendant d'un paramètre. Zeitschrift für Wahrscheinlichkeitstheorie und Verwandte Gebiete 45, 2 (1978), 109-133. 